\title{
Approximate Solutions of Hybrid Stochastic Pantograph Equations with Levy Jumps
}

\author{
Wei Mao ${ }^{1}$ and Xuerong $\mathrm{Mao}^{2}$ \\ ${ }^{1}$ School of Mathematics and Information Technology, Jiangsu Second Normal University, Nanjing 210013, China \\ ${ }^{2}$ Department of Statistics and Modelling Science, University of Strathclyde, Glasgow G1 1XH, UK \\ Correspondence should be addressed to Wei Mao; jsjysxx_mwzy@126.com
}

Received 17 March 2013; Accepted 9 May 2013

Academic Editor: Svatoslav Staněk

Copyright (c) 2013 W. Mao and X. Mao. This is an open access article distributed under the Creative Commons Attribution License, which permits unrestricted use, distribution, and reproduction in any medium, provided the original work is properly cited.

\begin{abstract}
We investigate a class of stochastic pantograph differential equations with Markovian switching and Levy jumps. We prove that the approximate solutions converge to the true solutions in $L^{2}$ sense as well as in probability under local Lipschitz condition and generalize the results obtained by Fan et al. (2007), Milošević and Jovanović (2011), and Marion et al. (2002) to cover a class of more general stochastic pantograph differential equations with jumps. Finally, an illustrative example is given to demonstrate our established theory.
\end{abstract}

\section{Introduction}

Stochastic delay differential equations (SDDEs) have come to play an important role in many branches of science and industry. Such models have been used with great success in a variety of application areas, including biology, epidemiology, mechanics, economics and finance. Similar to SDEs, an explicit solution can rarely be obtained for SDDEs. It is necessary to develop numerical methods and to study the properties of these methods. There are many results for the numerical solutions of SDDEs [1-12].

Recently, as a special case of SDDEs, a class of stochastic pantograph delay equations (SPEs) has been received a great deal of attention and various studies have been carried out on the convergence of SPEs [13-16]. However, all equations of the above-mentioned works are driven by white noise perturbations with continuous initial data, and white noise perturbations are not always appropriate to interpret real data in a reasonable way. In real phenomena, the state of stochastic pantograph delay system may be perturbed by abrupt pulses or extreme events. A more natural mathematical framework for these phenomena takes into account other than purely Brownian perturbations. In particular, we incorporate the Levy perturbations with jumps into stochastic pantograph delay system to model abrupt changes.
The study of the convergence of the numerical solutions to SDDEs with jumps is in its infancy [17-20], and there is no research on the numerical solutions to SPEs with Markovian switching and Levy jumps (SPEwMsLJs). In this paper, we study the strong convergence of the Euler method for a class of SPEs with Markovian switching and Levy jumps (SPEwMsLJs). SPEwMsLJs may be regarded as an extension of SPEs with Markovian switching and SPEs with Levy jumps. The main aim is to prove that the Euler approximate solutions converges to the true solutions for SPEwMsLJs in $L^{2}$ sense. On the other hand, we study the convergence in probability of the Euler approximate solutions to the true solutions under local Lipschitz condition and some additional conditions in term of Lyapunov-type functions. It should be pointed out that the proof for SPEwMsLJs is certainly not a straightforward generalization of that for SPEs and SPEwMs without Levy jumps. Although the way of analysis follows the ideas of [21], we need to develop several new techniques to deal with Levy jumps. Some known results in Fan et al. [14], Milošević and Jovanović [16], and Marion et al. [21] are generalized to cover a class of more general SPEwMsLJs.

The paper is organized as follows. In Section 2, we introduce some notations and hypotheses concerning (4), and the Euler methods is used to produce a numerical solutions. In Section 3, we establish some useful lemmas and prove that 
the approximate solutions converge to the true solutions of SPEwMsLJs in $L^{2}$ sense. By applying Theorem 4 , we study the convergence in probability of the approximate solutions to the true solutions in Section 4. Finally, we give an illustrative example in Section 5.

\section{Preliminaries and the Approximate Solution}

Let $(\Omega, \mathscr{F}, P)$ be a complete probability space with a filtration $\left(\mathscr{F}_{t}\right)_{t \geq 0}$ satisfying the usual condition; that is, the filtration $\left(\mathscr{F}_{t}\right)$ is continuous on the right and $\left(\mathscr{F}_{0}\right)$ contains all $P$-null sets. Let $\{W(t), t \geq 0\}$ be a $d$-dimensional Wiener process defined on the probability space $(\Omega, \mathscr{F}, P)$ adapted to the filtration $\left(\mathscr{F}_{t}\right)_{t \geq 0}$. Let $D\left([0, T], R^{n}\right)$ denote the family of function $f$ from $[0, T] \rightarrow R^{n}$ that are right continuous and have limits on the left. Also $D\left([0, T], R^{n}\right)$ is equipped with the norm $\|f\|=\sup _{0 \leq t \leq T}|f(t)|$, where $|\cdot|$ is the Euclidean norm in $R^{n}$; that is, $|x|=\sqrt{x^{\top} x}\left(x \in R^{n}\right)$. Let $T>0$, $P \geq 2$, and $\mathscr{L}^{P}\left([0, T] ; R^{n}\right)$ denote the family of all $R^{n}$-valued measurable $\left(\mathscr{F}_{t}\right)$-adapted processes $f=\{f(t)\}_{0 \leq t \leq T}$ such that $E \sup _{0 \leq t \leq T}|f(t)|^{P}<\infty$. Let $\left(R^{n}, \mathscr{B}\left(R^{n}\right)\right)$ be a measurable space and $\pi(d u)$ a $\sigma$-finite measure on it. Let $p=p(t)$, and let $t \in D_{p}$ be a stationary $\mathscr{F}_{t}$-Poisson point process on $R^{n}$ with characteristic measure $\pi$. Denote by $N(d t, d u)$ the Poisson counting measure associated with $p$; that is,

$$
N(t, A)=\sum_{s \in D_{p}, s \leq t} I_{A}(p(s)) .
$$

We refer to Mao [3] for the properties of a Wiener process and SDDEs and to Ikeda and Watanabe [22] for the details on Poisson point process.

Let $r(t), t \geq 0$, be a right-continuous Markov chain on the probability space $(\Omega, \mathscr{F}, P)$ taking values in a finite state space $S=\{1,2, \ldots, N\}$ with generator $\Gamma=\left(\gamma_{i j}\right)_{N \times N}$ given by

$$
\begin{aligned}
& P(r(t+\Delta)=j \mid r(t)=i) \\
& \quad= \begin{cases}\gamma_{i j} \Delta+\circ(\Delta), & \text { if } i \neq j, \\
1+\gamma_{i j} \Delta+\circ(\Delta), & \text { if } i=j,\end{cases}
\end{aligned}
$$

where $\Delta>0$. Here $\gamma_{i j} \geq 0$ is the transition rate from $i$ to $j$, $i \neq j$, while

$$
\gamma_{i i}=-\sum_{j \neq i} \gamma_{i j}
$$

We assume that Markov chain $r(\cdot)$ is independent of the Brownian motion $W(\cdot)$ and compensated Poisson random measure $\widetilde{N}(\cdot, \cdot)$. It is known that almost every sample path of $r(\cdot)$ is right-continuous step function with a finite number of simple jumps in any finite subinterval of $R_{+}$.
In this paper, we study the following hybrid stochastic pantograph equations with Levy jump:

$$
\begin{gathered}
d X(t)=f(X(t), X(q t), r(t)) d t \\
+g(X(t), X(q t), r(t)) d W(t) \\
+\int_{R^{n}} h(X(t), X(q t), r(t), u) \widetilde{N}(d t, d u), \\
X(0)=X_{0},
\end{gathered}
$$

where $0<q<1$ and

$$
\begin{gathered}
f: R^{+} \times R^{n} \times S \longrightarrow R^{n}, \\
g: R^{+} \times R^{n} \times S \longrightarrow R^{n \times m}, \\
h: R^{+} \times R^{n} \times S \times R^{n} \longrightarrow R^{n},
\end{gathered}
$$

$W(t)$ is a standard $m$-dimensional Brownian motion, and $\widetilde{N}(d t, d u)$ is the compensated Poisson random measure given by

$$
\widetilde{N}(d t, d u)=N(d t, d u)-\pi(d u) d t .
$$

Here $\pi(d u)$ is the Levy measure associated to $N$.

Let $C^{2}\left(R^{n} \times S, R_{+}\right)$denote the family of all nonnegative functions $V(x, i)$ on $R^{n} \times S$ which are continuously twice differentiable in $x$. For each $V \in C^{2}\left(R^{n} \times S, R_{+}\right)$, define an operator $L V$ from $R^{n} \times R^{n} \times S$ to $R$ by

$$
\begin{aligned}
& L V(x, y, i) \\
& \equiv V_{x}(x, i) f(x, y, i) \\
& \quad+\frac{1}{2} \operatorname{trace}\left[g^{\top}(x, y, i) V_{x x}(x, i) g(x, y, i)\right] \\
& \quad+\int_{R^{n}}[V(x+h(x, y, u), i)-V(x, i) \\
& \left.\quad-V_{x}(x, i) h(x, y, u)\right] \pi(d u) \\
& \quad+\sum_{j=1}^{N} \gamma_{i j} V(x, j),
\end{aligned}
$$

where

$$
\begin{gathered}
V_{x}(x, i)=\left(\frac{\partial V(x, i)}{\partial x_{1}}, \ldots, \frac{\partial V(x, i)}{\partial x_{n}}\right), \\
V_{x x}(x, i)=\left(\frac{\partial^{2} V(x, i)}{\partial x_{i} \partial x_{j}}\right)_{n \times n} .
\end{gathered}
$$

In order to define the Euler approximate solution of (4), we need the property of embedded discrete Markov chain. The following lemma [23] describes this property.

Lemma 1. For $h>0$ and $n \geq 0$, then $\left\{r_{n}^{h}, n=0,1,2, \ldots\right\}$ is a discrete Markov chain with the one-step transition probability matrix

$$
P(h)=\left(P_{i j}(h)\right)_{N \times N}=e^{h \Gamma}
$$


Given a step size $h>0$, the discrete Markov chain $\left\{r_{n}^{h}, n=0,1,2, \ldots\right\}$ can be simulated as follows (see Mao and Yuan [24]). Let $r(0)=i_{0}$ and generate a random number $\zeta_{1}$ which is uniformly distributed in $[0,1]$. If $\zeta_{1}=1$, then let $r_{1}^{h}=i_{1}=N$ or otherwise find the unique integer $i_{1} \in S$ for

$$
\sum_{j=1}^{i_{1}-1} P_{i_{0}, j}(h) \leq \zeta_{1}<\sum_{j=1}^{i_{1}} P_{i_{0}, j}(h)
$$

and let $r_{1}^{h}=i_{1}$, where we set $\sum_{i=1}^{0} P_{i_{0}, j}(h)=0$ as usual. Generate independently a new random number $\zeta_{2}$ which is again uniformly distributed in $[0,1]$. If $\zeta_{2}=1$ then let $r_{2}^{h}=i_{2}=N$ or otherwise find the unique integer $i_{2} \in S$ for

$$
\sum_{j=1}^{i_{2}-1} P_{i_{1}, j}(h) \leq \zeta_{2}<\sum_{j=1}^{i_{2}} P_{i_{1}, j}(h)
$$

and let $r_{2}^{h}=i_{2}$. Repeating this procedure, a trajectory of $\left\{r_{n}^{h}, n=0,1,2, \ldots\right\}$ can be generated. This procedure can be carried out independently to obtain more trajectories.

Now we define the Euler approximate solution to (4) with discrete Markov chain $\left\{r_{n}^{h}=r(n h), n=0,1,2, \ldots\right\}$. For system (4), the discrete approximation is given by the iterative scheme

$$
\begin{aligned}
Y_{n+1}= & Y_{n}+f\left(Y_{n}, Y_{[q n]}, r_{n}^{h}\right) h \\
& +g\left(Y_{n}, Y_{[q n]}, r_{n}^{h}\right) \Delta W_{n} \\
& +\int_{R^{n}} h\left(Y_{n}, Y_{[q n]}, r_{n}^{h}, u\right) \widetilde{N}(h, d u),
\end{aligned}
$$

with initial value $Y_{0}=X(0)$, and $[u]$ represents the integer part of $u$. Here $t_{n}=n h$ for $n \geq 0$. We have $Y_{n} \approx X\left(t_{n}\right)$, $Y_{[q n]} \approx X\left(q t_{n}\right), \Delta W_{n}=W\left(t_{n+1}\right)-W\left(t_{n}\right)$, and $\widetilde{N}(h, d u)=$ $\widetilde{N}\left(t_{n+1}, d u\right)-\widetilde{N}\left(t_{n}, d u\right)$.

Let us introduce the following notations:

$$
Z_{1}(t)=Y_{n}, \quad Z_{2}(t)=Y_{[q n]}, \quad \bar{r}(t)=r_{n}^{h}
$$

for $t \in\left[t_{n}, t_{n+1}\right)$. Then we define the continuous Euler approximate solution as follows:

$$
\begin{aligned}
Y(t)= & Y(0)+\int_{0}^{t} f\left(Z_{1}(s), Z_{2}(s), \bar{r}(s)\right) d s \\
& +\int_{0}^{t} g\left(Z_{1}(s), Z_{2}(s), \bar{r}(s)\right) d W(s) \\
& +\int_{0}^{t} \int_{R^{n}} h\left(Z_{1}(s), Z_{2}(s), \bar{r}(s), u\right) \widetilde{N}(d s, d u), \\
& 0 \leq t \leq T,
\end{aligned}
$$

which interpolates the discrete approximation (7).

In order to establish the strong convergence theorem, we suppose the following assumptions are satisfied.
Assumption 2. For each $i \in S$ and $u \in R^{n}$,

$$
|f(0,0, i)|^{2}=|g(0,0, i)|^{2}=\int_{R^{n}}|h(0,0, i, u)|^{2} \pi(d u)=0 .
$$

Assumption 3. For every $d \geq 1$, there exists a positive constant $K_{d}$ such that for all $x_{1}, y_{1}, x_{2}, y_{2}, u \in R^{n}$ and $i \in S$, $\left|x_{1}\right| \vee$ $\left|y_{1}\right| \vee\left|x_{2}\right| \vee\left|y_{2}\right| \leq d$,

$$
\begin{aligned}
&\left|f\left(x_{1}, y_{1}, i\right)-f\left(x_{2}, y_{2}, i\right)\right|^{2} \\
& \vee\left|g\left(x_{1}, y_{1}, i\right)-g\left(x_{2}, y_{2}, i\right)\right|^{2} \\
& \quad \vee \int_{R^{n}}\left|h\left(x_{1}, y_{1}, i, u\right)-h\left(x_{2}, y_{2}, i, u\right)\right|^{2} \pi(d u) \\
& \quad \leq K_{d}\left(\left|x_{1}-x_{2}\right|^{2}+\left|y_{1}-y_{2}\right|^{2}\right) .
\end{aligned}
$$

\section{Strong Convergence of Numerical Solutions}

In this section, we will prove that the Euler approximate solutions converge to the true solutions in $L^{2}$ sense under the local Lipschitz condition.

Theorem 4. If Assumptions 2 and 3 hold, then the Euler approximate solutions converge to the true solutions of (4) in $L^{2}$ sense with order $1 / 2$; that is, there exists a positive constant $C_{d}$ such that

$$
E\left[\sup _{0 \leq t \leq T \wedge \tau_{d}}|X(t)-Y(t)|^{2}\right] \leq C_{d}[h+\circ(h)],
$$

where $\theta_{d}=\inf \{t \in[0, T]:|X(t)| \geq d\}$ and $\rho_{d}=\inf \{t \in$ $[0, T]:|Y(t)| \geq d\}$, and let $\tau_{d}=\theta_{d} \wedge \rho_{d}$.

The proof of Theorem 4 is very technical, so we present some useful lemmas.

Lemma 5. Under Assumptions 2 and 3 , for any $t \in[0, T]$ and $P \geq 2$, there exists a positive constant $C_{1}(d)$ such that

$$
E \int_{0}^{t}\left|Y\left(s \wedge \tau_{d}\right)-Z_{1}\left(s \wedge \tau_{d}\right)\right|^{P} d s \leq C_{1}(d) h^{P / 2} T,
$$

where $C_{1}(d)$ is a positive constant independent of the step size $h$.

Proof. For any $t \in\left[0, T \wedge \tau_{d}\right]$, there exists an integer $n$ such that $t \in[n h,(n+1) h)$. Then

$$
\begin{aligned}
Y(t)- & Z_{1}(t) \\
= & Y(t)-Y_{n} \\
= & \int_{n h}^{t} f\left(Z_{1}(s), Z_{2}(s), \bar{r}(s)\right) d s \\
& +\int_{n h}^{t} g\left(Z_{1}(s), Z_{2}(s), \bar{r}(s)\right) d W(s) \\
& +\int_{n h}^{t} \int_{R^{n}} h\left(Z_{1}(s), Z_{2}(s), \bar{r}(s), u\right) \widetilde{N}(d s, d u) .
\end{aligned}
$$


Using the inequality $|a+b+c|^{P} \leq 3^{P-1}\left[|a|^{P}+3|b|^{P}+3|c|^{P}\right]$, we get

$$
\begin{aligned}
& E\left[\sup _{n h \leq t \leq(n+1) h}\left|Y(t)-Z_{1}(t)\right|^{P}\right] \\
& \leq 3^{P-1}\left[E \left(\sup _{n h \leq t \leq(n+1) h} \mid \int_{n h}^{t} f\left(Z_{1}(s),\right.\right.\right. \\
& \left.\left.+Z_{2}(s), \bar{r}(s)\right)\left.d s\right|^{P}\right) \\
& +3^{P-1} E \\
& \times\left(\sup _{n h \leq t \leq(n+1) h} \mid \int_{n h}^{t} g\left(Z_{1}(s),\right.\right. \\
& +3^{P-1} E\left(\sup _{n h \leq t \leq(n+1) h}\left|\int_{n h}^{t} \int_{R^{n}} h\left(Z_{1}(s), \bar{r}(s)\right) d W(s)\right|^{P}\right) \\
& \left.\quad Z_{2}(s), \bar{r}(s), u\right) \\
& \left.\left.\times\left.\widetilde{N}(d s, d u)\right|^{P}\right)\right] .
\end{aligned}
$$

By the Hölder inequality and Assumptions 2 and 3, we have

$$
\begin{aligned}
& E\left(\left.\sup _{n h \leq t \leq(n+1) h}|| \int_{n h}^{t} f\left(Z_{1}(s), Z_{2}(s), \bar{r}(s)\right) d s\right|^{P}\right) \\
& \leq E\left(\left|\int_{n h}^{(n+1) h} f\left(Z_{1}(s), Z_{2}(s), \bar{r}(s)\right) d s\right|^{P}\right) \\
& \leq h^{P-1} E \int_{n h}^{(n+1) h}\left|f\left(Z_{1}(s), Z_{2}(s), \bar{r}(s)\right)\right|^{P} d s \\
& \leq h^{P-1} E \int_{n h}^{(n+1) h}\left[\left|f\left(Z_{1}(s), Z_{2}(s), \bar{r}(s)\right)\right|^{2}\right]^{P / 2} d s \\
& \leq h^{P-1} E \int_{n h}^{(n+1) h}\left[2 \mid f\left(Z_{1}(s), Z_{2}(s), \bar{r}(s)\right)\right. \\
& \quad-f(0,0),\left.\bar{r}(s)\right|^{2} \\
& \leq h^{P-1} E \int_{n h}^{(n+1) h}\left[2 K_{d}\left(\left|Z_{1}(s)\right|^{2}+\left|Z_{2}(s)\right|^{2}\right)\right]^{P / 2} d s \\
& \leq h^{P-1} 2^{P-1} K_{d}^{P / 2} E \int_{n h}^{(n+1) h}\left(\left|Z_{1}(s)\right|^{P}+\left|Z_{2}(s)\right|^{P}\right) d s .
\end{aligned}
$$

By the definition of $\tau_{d}$, we have $|Y(t)|<d, t \in\left[0, \tau_{d} \wedge T\right]$. so we get that $\left|Z_{1}(t)\right|^{P}<d^{P},\left|Z_{2}(t)\right|^{P}<d^{P}$, and

$$
\begin{gathered}
E\left(\sup _{n h \leq t \leq(n+1) h}\left|\int_{n h}^{t} f\left(Z_{1}(s), Z_{2}(s), \bar{r}(s)\right) d s\right|^{P}\right) \\
\leq 2^{P} d^{P} K_{d}^{P / 2} h^{P} .
\end{gathered}
$$

By using the Burkholder-Davis-Gundy inequality and Assumptions 2 and 3, we have

$$
\begin{aligned}
& E\left(\sup _{n h \leq t \leq(n+1) h}\left|\int_{n h}^{t} g\left(Z_{1}(s), Z_{2}(s), \bar{r}(s)\right) d W(s)\right|^{P}\right) \\
& \leq C_{P} E\left[\int_{n h}^{(n+1) h}\left|g\left(Z_{1}(s), Z_{2}(s), \bar{r}(s)\right)\right|^{2} d s\right]^{P / 2} \\
& \leq C_{P} h^{(P / 2)-1} E\left[\int_{n h}^{(n+1) h}\left|g\left(Z_{1}(s), Z_{2}(s), \bar{r}(s)\right)\right|^{P} d s\right] \\
& \leq C_{p^{2}} 2^{P} d^{P} K_{d}^{P / 2} h^{P / 2}, \\
& E\left(\sup _{n h \leq t \leq(n+1) h}\left|\int_{n h}^{t} \int_{R^{n}} h\left(Z_{1}(s), Z_{2}(s), \bar{r}(s), u\right) \widetilde{N}(d s, d u)\right|^{P}\right) \\
& \leq C_{P} E\left[\int_{n h}^{(n+1) h} \int_{R^{n}}\left|h\left(Z_{1}(s), Z_{2}(s), \bar{r}(s), u\right)\right|^{2} \pi(d u) d s\right]^{P / 2} \\
& \leq C_{P} h^{(P / 2)-1} E \\
& \quad \times \int_{n h}^{(n+1) h}\left[\int_{R^{n}}\left|h\left(Z_{1}(s), Z_{2}(s), \bar{r}(s), u\right)\right|^{2} \pi(d u)\right]^{P / 2} d s \\
& \leq C_{P} h^{(P / 2)-1} E \\
& \quad \times \int_{n h}^{(n+1) h}\left\{\int _ { R ^ { n } } \left[2 \mid h\left(Z_{1}(s), Z_{2}(s), \bar{r}(s), u\right)\right.\right. \\
& \leq C_{P} h^{(P / 2)-1} E \int_{n h}^{(n+1) h}\left[2 K_{d}\left(\left|Z_{1}(s)\right|^{2}+\left|Z_{2}(s)\right|^{2}\right)\right]^{P / 2} d s \\
& \leq C_{p} 2^{P} d^{P} K_{d}^{P / 2} h^{P / 2} . \\
& \quad-\left.h(0,0, \bar{r}(s), u)\right|^{2}
\end{aligned}
$$

Combing (22)-(24) together, we have

$$
\begin{aligned}
& E\left|Y(t)-Z_{1}(t)\right|^{P} \\
& \quad \leq 3^{P-1}\left[2^{P} d^{P} K_{d}^{P / 2} h^{P}+2 C_{p} 2^{P} d^{P} K_{d}^{P / 2} h^{P / 2}\right], \\
& E \int_{0}^{t \wedge \tau_{d}}\left|Y(s)-Z_{1}(s)\right|^{P} d s \leq C_{1}(d) h^{P / 2} T,
\end{aligned}
$$

where $C_{1}(d)=3^{P-1}\left[2^{P} d^{P} K_{d}^{P / 2}+2 C_{p} 2^{P} d^{P} K_{d}^{P / 2}\right]$. The proof is complete. 
Lemma 6. Under Assumptions 2 and 3, for any $t \in[0, T]$ and $P \geq 2$, then

$$
E \int_{0}^{t}\left|Y\left(q\left(s \wedge \tau_{d}\right)\right)-Z_{2}\left(s \wedge \tau_{d}\right)\right|^{P} d s \leq C_{2}(d) h^{P / 2} T
$$

where $C_{2}(d)$ is a positive constant independent of the stepsize $h$.

The proof of this lemma is similar to that of Lemma 5.

Lemma 7. Under Assumptions 2 and 3, for any $t \in[0, T]$ and $P \geq 2$, then

$$
\begin{gathered}
E \int_{0}^{t} \mid f\left(Z_{1}\left(s \wedge \tau_{d}\right), Z_{2}\left(s \wedge \tau_{d}\right), r\left(s \wedge \tau_{d}\right)\right) \\
-\left.f\left(Z_{1}\left(s \wedge \tau_{d}\right), Z_{2}\left(s \wedge \tau_{d}\right), \bar{r}\left(s \wedge \tau_{d}\right)\right)\right|^{P} d s \\
\vee E \int_{0}^{t} \mid g\left(Z_{1}\left(s \wedge \tau_{d}\right), Z_{2}\left(s \wedge \tau_{d}\right), r\left(s \wedge \tau_{d}\right)\right) \\
\quad-\left.g\left(Z_{1}\left(s \wedge \tau_{d}\right), Z_{2}\left(s \wedge \tau_{d}\right), \bar{r}\left(s \wedge \tau_{d}\right)\right)\right|^{P} d s \\
\quad \int_{0}^{t} \int_{R^{n}} \mid h\left(Z_{1}\left(s \wedge \tau_{d}\right), Z_{2}\left(s \wedge \tau_{d}\right), r\left(s \wedge \tau_{d}\right), u\right) \\
\quad-\left.h\left(Z_{1}\left(s \wedge \tau_{d}\right), Z_{2}\left(s \wedge \tau_{d}\right), \bar{r}\left(s \wedge \tau_{d}\right), u\right)\right|^{P} \\
\times \pi(d u) d s \leq C_{3}(d)\left[h^{P / 2}+\circ\left(h^{P / 2}\right)\right]
\end{gathered}
$$

where $C_{3}(d)$ is a positive constant independent of the stepsize $h$.

The proof of this lemma is similar to that of $[16,24]$.

Proof of Theorem 4. Combining (4) and (14), one has

$$
\begin{aligned}
& X(t)-Y(t) \\
& =\int_{0}^{t}[f(X(s), X(q s), r(s)) \\
& \left.\quad-f\left(Z_{1}(s), Z_{2}(s), \bar{r}(s)\right)\right] d s \\
& +\int_{0}^{t}[g(X(s), X(q s), r(s)) \\
& +\int_{0}^{t} \int_{R^{n}}[h(X(s), X(q s), r(s), u) \\
& \left.\quad-g\left(Z_{1}(s), Z_{2}(s), \bar{r}(s)\right)\right] d W(s) \\
& \left.\quad h\left(Z_{1}(s), Z_{2}(s), \bar{r}(s), u\right)\right] \widetilde{N}(d s, d u) .
\end{aligned}
$$

Then applying the generalized Itô's formula, we can show that

$$
\begin{aligned}
& |X(t)-Y(t)|^{2} \\
& =2 \int_{0}^{t}|X(s)-Y(s)| \\
& \quad \times \mid f(X(s), X(q s), r(s)) \\
& +\int_{0}^{t}\left|g(X(s), X(q s), r(s))-g\left(Z_{1}(s), Z_{2}(s), \bar{r}(s)\right)\right|^{2} d s \\
& \left.+\int_{0}^{t} \int_{R^{n}} \mid h(x), Z_{2}(s), \bar{r}(s)\right) \mid d s \\
& +2 \int_{0}^{t}|X(s)-Y(s)| \\
& +\int_{0}^{t} \int_{R^{n}}\left|h\left(Z_{1}(s), Z_{2}(s), \bar{r}(s), u\right)\right|^{2} \pi(d u) d s \\
& \quad \times \mid g(s), r(s), X(q s), r(s)) \\
& \quad-\left.h\left(Z_{1}(s), Z_{2}(s), \bar{r}(s), u\right)\right|^{2} \widetilde{N}(d s, d u) \\
& \quad-g\left(Z_{1}(s), Z_{2}(s), \bar{r}(s)\right) \mid d W(s) \\
& \left.+\int_{1}^{t}(s), X(q s), r(s), u\right)
\end{aligned}
$$

Hence, for any $t \in[0, T]$, we get

$$
\begin{aligned}
& E \sup _{0 \leq t \leq T \wedge \tau_{d}}|X(t)-Y(t)|^{2} \\
& \leq E \int_{0}^{T \wedge \tau_{d}}|X(s)-Y(s)|^{2} d s \\
& +E \int_{0}^{\tau_{d} \wedge T} \mid f(X(s), X(q s), r(s)) \\
& +E \int_{0}^{\tau_{d} \wedge T} \mid g(X(s), X(q s), r(s)) \\
& +E \int_{0}^{\tau_{d} \wedge T} \int_{R^{n}} \mid h(X(s), X(q s), r(s), u)
\end{aligned}
$$




$$
\begin{array}{r}
-\left.h\left(Z_{1}(s), Z_{2}(s), \bar{r}(s), u\right)\right|^{2} \pi(d u) d s \\
+2 E \sup _{0 \leq t \leq \tau_{d} \wedge T} \int_{0}^{t}\left|X\left(s \wedge \tau_{d}\right)-Y\left(s \wedge \tau_{d}\right)\right| \\
\times \mid g\left(X\left(s \wedge \tau_{d}\right), X\left(q\left(s \wedge \tau_{d}\right)\right), r(s)\right) \\
-g\left(Z_{1}(s), Z_{2}(s), \bar{r}(s)\right) \mid d W(s) \\
+E \sup _{0 \leq t \leq \tau_{d} \wedge T} \int_{0}^{t} \int_{R^{n}}[2|X(s)-Y(s)| \\
\times \mid h(X(s), X(q s), r(s), u) \\
\left.-h\left(Z_{1}(s), Z_{2}(s), \bar{r}(s), u\right) \mid\right] \\
\times \widetilde{N}(d s, d u) \\
+E \sup _{0 \leq t \leq \tau_{d} \wedge T} \int_{0}^{t} \int_{R^{n}} \mid h(X(s), X(q s), r(s), u) \\
-\left.h\left(Z_{1}(s), Z_{2}(s), \bar{r}(s), u\right)\right|^{2} \\
\times \widetilde{N}(d s, d u) .
\end{array}
$$

By Assumption 3 and Lemmas 5-7, we have

$$
\begin{aligned}
& E \int_{0}^{\tau_{d} \wedge T} \mid f(X(s), X(q s), r(s)) \\
& -\left.f\left(Z_{1}(s), Z_{2}(s), \bar{r}(s)\right)\right|^{2} d s \\
& \leq 2 E \int_{0}^{\tau_{d} \wedge T} \mid f(X(s), X(q s), r(s)) \\
& -\left.f\left(Z_{1}(s), Z_{2}(s), r(s)\right)\right|^{2} d s \\
& +2 E \int_{0}^{\tau_{d} \wedge T} \mid f\left(Z_{1}(s), Z_{2}(s), r(s)\right) \\
& -\left.f\left(Z_{1}(s), Z_{2}(s), \bar{r}(s)\right)\right|^{2} d s \\
& \leq 2 K_{d} E \int_{0}^{\tau_{d} \wedge T}\left(\left|X(s)-Z_{1}(s)\right|^{2}\right. \\
& \left.+\left|X(q s)-Z_{2}(s)\right|^{2}\right) d s+2 C_{3}(d) h \\
& \leq 2 K_{d} E \\
& \times \int_{0}^{\tau_{d} \wedge T}\left(2|X(s)-Y(s)|^{2}+\left|Y(s)-Z_{1}(s)\right|^{2}\right) \\
& +\left(2|X(q s)-Y(q s)|^{2}+2\left|Y(q s)-Z_{2}(s)\right|^{2}\right) d s \\
& +2 C_{3}(d)[h+\circ(h)] \\
& \leq 4 K_{d}\left[C_{1}(d)+C_{2}(d)\right] h T+2 C_{3}(d)[h+\circ(h)] \\
& +8 K_{d} \int_{0}^{\tau_{d} \wedge T} E \sup _{0 \leq u \leq s}|X(u)-Y(u)|^{2} d s .
\end{aligned}
$$

Similarly, by Assumption 3 and Lemmas 5-7, we obtain

$$
\begin{aligned}
& E \int_{0}^{\tau_{d} \wedge T} \mid g(X(s), X(q s), r(s)) \\
& -\left.g\left(Z_{1}(s), Z_{2}(s), \bar{r}(s)\right)\right|^{2} d s \\
& \leq 4 K_{d}\left[C_{1}(d)+C_{2}(d)\right] h T+2 C_{3}(d)[h+\circ(h)] \\
& +8 K_{d} \int_{0}^{\tau_{d} \wedge T} E \sup _{0 \leq u \leq s}|X(u)-Y(u)|^{2} d s, \\
& E \int_{0}^{\tau_{d} \wedge T} \int_{R^{n}} \mid h(X(s), X(q s), r(s), u) \\
& -\left.h\left(Z_{1}(s), Z_{2}(s), \bar{r}(s), u\right)\right|^{2} \pi(d u) d s \\
& \leq 4 K_{d}\left[C_{1}(d)+C_{2}(d)\right] h T+2 C_{3}(d)[h+\circ(h)] \\
& +8 K_{d} \int_{0}^{\tau_{d} \wedge T} E \sup _{0 \leq u \leq s}|X(u)-Y(u)|^{2} d s .
\end{aligned}
$$

On the other hand, by the Burkholder-Davis-Gundy inequality, Young's inequality, and Lemmas $5-7$, we have for any $\varepsilon_{1}>0$

$$
\begin{aligned}
E \sup _{0 \leq t \leq \tau_{d} \wedge T} \int_{0}^{t}|X(s)-Y(s)| & \\
& \times \mid g(X(s), X(q s), r(s)) \\
& -g\left(Z_{1}(s), Z_{2}(s), \bar{r}(s)\right) \mid d W(s) \\
\leq & 3 E\left[\sup _{0 \leq t \leq \tau_{d} \wedge T}|X(t)-Y(t)|\right. \\
& \times\left(\int_{0}^{\tau_{d} \wedge T} \mid g(X(t), X(q t), r(t))\right. \\
\leq 3\left[\varepsilon_{1} E \sup _{0 \leq t \leq \tau_{d} \wedge T}|X(t)-Y(t)|^{2}\right]^{1 / 2} & \left.\left.-\left.g\left(Z_{1}(t), Z_{2}(t), \bar{r}(t)\right)\right|^{2} d t\right)\right]^{1 / 2} \\
\times & {\left[\frac { 1 } { \varepsilon _ { 1 } } E \left(\int_{0}^{\tau_{d} \wedge T} \mid g(X(t), X(q t), r(t))\right.\right.} \\
\leq & 6 \varepsilon_{1} E \sup _{0 \leq t \leq \tau_{d} \wedge T}|X(t)-Y(t)|^{2} \\
+\frac{6}{\varepsilon_{1}} E \int_{0}^{\tau_{d} \wedge T} \mid g(X(t), X(q t), r(t)) & -\left.g\left(Z_{1}(t), Z_{2}(t), \bar{r}(t)\right)\right|^{2} d t
\end{aligned}
$$




$$
\begin{aligned}
\leq & 6 \varepsilon_{1} E \sup _{0 \leq t \leq \tau_{d} \wedge T}|X(t)-Y(t)|^{2} \\
& +\frac{24}{\varepsilon_{1}} K_{d}\left[C_{1}(d)+C_{2}(d)\right] h T \\
& +\frac{12}{\varepsilon_{1}} C_{3}(d)[h+\circ(h)] \\
& +\frac{48}{\varepsilon_{1}} K_{d} \int_{0}^{\tau_{d} \wedge T} E \sup _{0 \leq u \leq s}|X(u)-Y(u)|^{2} d s .
\end{aligned}
$$

We have for any $\varepsilon_{2}>0$

$$
\begin{aligned}
& E \sup _{0 \leq t \leq \tau_{d} \wedge T} \int_{0}^{t} \int_{R^{n}}[2|X(s)-Y(s)| \mid h(X(s), X(q s), r(s), u) \\
& \left.-h\left(Z_{1}(s), Z_{2}(s), \bar{r}(s), u\right) \mid\right] \widetilde{N}(d s, d u) \\
& \leq C E\left(\sum_{t \in D_{p}, t \leq \tau \wedge T}|X(t)-Y(t)|^{2}\right. \\
& \times \mid h\left(X(t), X(q t), r(t), p_{t}\right) \\
& \left.-\left.h\left(Z_{1}(t), Z_{2}(t), \bar{r}(t), p_{t}\right)\right|^{2}\right)^{1 / 2} \\
& \leq C E \sup _{0 \leq t \leq \tau_{d} \wedge T}|X(t)-Y(t)| E \\
& \times\left(\sum_{t \in D_{p}, t \leq \tau_{d} \wedge T} \mid h\left(X(t), X(q t), r(t), p_{t}\right)\right. \\
& \left.-\left.h\left(Z_{1}(t), Z_{2}(t), \bar{r}(t), p_{t}\right)\right|^{2}\right)^{1 / 2} \\
& \leq C E\left[\varepsilon_{2} \sup _{0 \leq t \leq \tau_{d} \wedge T}|X(t)-Y(t)|^{2}\right]^{1 / 2} \\
& \times E\left[\frac{1}{\varepsilon_{2}} \sum_{t \in D_{p}, t \leq \tau_{d} \wedge T} \mid h\left(X(t), X(q t), r(t), p_{t}\right)\right. \\
& \left.-\left.h\left(Z_{1}(t), Z_{2}(t), \bar{r}(t), p_{t}\right)\right|^{2}\right]^{1 / 2} \\
& \leq C \varepsilon_{2} E \sup _{0 \leq t \leq \tau_{d} \wedge T}|X(t)-Y(t)|^{2} \\
& +\frac{C}{\varepsilon_{2}} E\left(\sum_{t \in D_{p}, t \leq \tau_{d} \wedge T} \mid h\left(X(t), X(q t), r(t), p_{t}\right)\right. \\
& \left.-\left.h\left(Z_{1}(t), Z_{2}(t), \bar{r}(t), p_{t}\right)\right|^{2}\right)
\end{aligned}
$$

$$
\begin{aligned}
& \leq C \varepsilon_{2} E \sup _{0 \leq t \leq \tau_{d} \wedge T}|X(t)-Y(t)|^{2} \\
& +\frac{C}{\varepsilon_{2}} E \int_{0}^{\tau_{d} \wedge T} \int_{R^{n}} \mid h(X(t), X(q t), r(t), u) \\
& \quad-\left.h\left(Z_{1}(t), Z_{2}(t), \bar{r}(t), u\right)\right|^{2} \pi(d u) d t \\
& \leq C \varepsilon_{2} E \sup _{0 \leq t \leq \tau_{d} \wedge T}|X(t)-Y(t)|^{2} \\
& +\frac{4 C}{\varepsilon_{2}} K_{d}\left[C_{1}(d)+C_{2}(d)\right] h T+\frac{2 C}{\varepsilon_{2}} C_{3}(d)[h+\circ(h)] \\
& +\frac{8 C}{\varepsilon_{2}} K_{d} \int_{0}^{\tau_{d} \wedge T} E \sup _{0 \leq u \leq s}|X(u)-Y(u)|^{2} d s,
\end{aligned}
$$

where $C$ is some constant that may change from line to line. Similar, we have

$$
\begin{aligned}
& E \sup _{0 \leq t \leq \tau_{d} \wedge T} \int_{0}^{t} \int_{R^{n}} \mid h(X(s), X(q s), r(s), u) \\
& -\left.h\left(Z_{1}(s), Z_{2}(s), \bar{r}(s), u\right)\right|^{2} \widetilde{N}(d s, d u) \\
& \leq C E\left(\sum_{t \in D_{p}, t \leq \tau_{d} \wedge T} \mid h\left(X(t), X(q t), r(t), p_{t}\right)\right. \\
& \left.-\left.h\left(Z_{1}(t), Z_{2}(t), \bar{r}(t), p_{t}\right)\right|^{4}\right)^{1 / 2} \\
& \leq C E\left(\sum_{s \in D_{p}, s \leq \tau_{d} \wedge T} \mid h\left(X(t), X(q t), r(t), p_{t}\right)\right. \\
& \left.-\left.h\left(Z_{1}(t), Z_{2}(t), \bar{r}(t), p_{t}\right)\right|^{2}\right) \\
& \leq C E \int_{0}^{\tau_{d} \wedge T} \int_{R^{n}} \mid h(X(t), X(q t), r(t), u) \\
& -\left.h\left(Z_{1}(t), Z_{2}(t), \bar{r}(t), u\right)\right|^{2} \pi(d u) d t \\
& \leq 4 C K_{d}\left[C_{1}(d)+C_{2}(d)\right] h T+2 C C_{3}(d)[h+\circ(h)] \\
& +8 C K_{d} \int_{0}^{\tau_{d} \wedge T} E \sup _{0 \leq u \leq s}|X(u)-Y(u)|^{2} d s .
\end{aligned}
$$


Substituting (31)-(36) into (30), we obtain that

$$
\begin{aligned}
& E\left[\sup _{0 \leq t \leq \tau_{d} \wedge T}|X(t)-Y(t)|^{2}\right] \\
& \leq\left(6 \varepsilon_{1}+C \varepsilon_{2}\right) E\left[\sup _{0 \leq t \leq \tau_{d} \wedge T}|X(t)-Y(t)|^{2}\right] \\
&+\left(10+\frac{24}{\varepsilon_{1}}+2 C \frac{1+\varepsilon_{2}}{\varepsilon_{2}}\right) K_{d}\left[C_{1}(d)+C_{2}(d)\right] T h \\
&+\left(4+\frac{12}{\varepsilon_{1}}\right) C_{3}(d)[h+\circ(h)] \\
&+\left[1+\left(20+\frac{48}{\varepsilon_{1}}+4 C \frac{1+\varepsilon_{2}}{\varepsilon_{2}}\right) K_{d}\right] \\
& \times \int_{0}^{T} E \sup _{0 \leq u \leq \tau \wedge s}|X(u)-Y(u)|^{2} d s .
\end{aligned}
$$

By choosing $\varepsilon_{1}>0, \varepsilon_{2}>0$ sufficiently small and letting $6 \varepsilon_{1}+$ $C \varepsilon_{2}=2 / 3$, we have

$$
\begin{aligned}
& E\left[\sup _{0 \leq t \leq \tau \wedge T}|X(t)-Y(t)|^{2}\right] \\
& \leq 3\left(10+\frac{24}{\varepsilon_{1}}+2 C \frac{1+\varepsilon_{2}}{\varepsilon_{2}}\right) K_{d}\left[C_{1}(d)+C_{2}(d)\right] T h \\
&+3\left(4+\frac{12}{\varepsilon_{1}}\right) C_{3}(d)[h+\circ(h)] \\
&+3\left[1+\left(20+\frac{48}{\varepsilon_{1}}+4 C \frac{1+\varepsilon_{2}}{\varepsilon_{2}}\right) K_{d}\right] \\
& \times \int_{0}^{T} E \sup _{0 \leq u \leq \tau \wedge s}|X(u)-Y(u)|^{2} d s .
\end{aligned}
$$

Therefore, we apply Gronwall's inequality to get

$$
\begin{aligned}
& E\left[\sup _{0 \leq t \leq \tau \wedge T}|X(t)-Y(t)|^{2}\right] \\
& \leq\left\{3\left(10+\frac{24}{\varepsilon_{1}}+2 C \frac{1+\varepsilon_{2}}{\varepsilon_{2}}\right) K_{d}\left[C_{1}(d)+C_{2}(d)\right] T h\right. \\
& \left.+3\left(4+\frac{12}{\varepsilon_{1}}\right) C_{3}(d)[h+\circ(h)]\right\} e^{3\left[1+\left(20+\left(48 / \varepsilon_{1}\right)+4 C\left(\left(1+\varepsilon_{2}\right) / \varepsilon_{2}\right)\right) K_{d}\right]} .
\end{aligned}
$$

This completes the proof.

Remark 8. Under the local Lipschitz condition, Theorem 4 not only tells us the strong convergence of the approximate solutions to the true solutions but also tells us the rate of the convergence with order $1 / 2$ by (39).

Remark 9. When $r(t) \equiv 0$ or $h \equiv 0$, (4) reduces to which was studied by Fan et al. [14], Xiao and Zhang [15], and Milošević and Jovanović [16]. Our results in the present paper generalized and improved the results in [14-16].

\section{Convergence of Numerical Solutions in Probability}

In this section, by applying Theorem 4, we will show the convergence in probability of the approximate solutions to the true solutions under local Lipschitz condition. Before we give the convergence theorem, we need some additional conditions based on Lyapunov-type functions.

Assumption 10. For $x \in R^{n}$ and $i \in S$, there exist a positive function $V \in C^{2}\left(R^{n} \times S ; R^{+}\right), K>0$, and two constants $\lambda_{1}>$ $\lambda_{2} \geq 0$ such that

$$
\begin{gathered}
\lim _{|x| \rightarrow \infty} V(x, i)=\infty, \\
L V(x, y, i) \leq K\left[1-\lambda_{1} V(x, i)+\lambda_{2} V(y, i)\right] .
\end{gathered}
$$

Assumption 11. There exists a positive constant $L_{d}$ such that, for all $x, y \in R^{n}$ and $i \in S$ with $|x| \vee|y| \leq d$,

$$
\begin{aligned}
& |V(x, i)-V(y, i)| \vee\left|V_{x}(x, i)-V_{x}(y, i)\right| \\
& \quad \vee\left|V_{x x}(x, i)-V_{x x}(y, i)\right| \leq L_{d}|x-y| .
\end{aligned}
$$

Now, let us state our convergence theorem.

Theorem 12. Let the assumptions of Theorem 4 hold. Also assume that there exists a $C^{2}$ function $V: R^{n} \times S \rightarrow R_{+}$satisfying (40)-(42). Then the Euler approximate solutions converges to the true solutions of (4) in the sense of the probability.

That is,

$$
\lim _{h \rightarrow 0} \sup _{0 \leq t \leq T}|Y(t)-X(t)|^{2}=0, \quad \text { in probability. }
$$

Proof. The proof is rather technical, and we divide it into three steps.

Step 1. We assume the existence of the nonnegative Lyapunov function $V(x, i)$ satisfying (40). Applying the Itô's formula, $V(X(t), r(t))$ yields

$$
\begin{aligned}
& d V(X(t), r(t)) \\
& =L V(X(t), X(q t), r(t)) d t \\
& +V_{x}(X(t), r(t)) g(X(t), X(q t), r(t)) d W(t) \\
& +\int_{R^{n}}[V(X(t)+h(X(t), X(q t), r(t), u), r(t)) \\
& \quad-V(X(t), r(t))] \widetilde{N}(d t, d u) .
\end{aligned}
$$

Integrating from 0 to $t \wedge \theta_{d}$ and taking expectations gives

$$
\begin{aligned}
E V & \left(X\left(t \wedge \theta_{d}\right), r\left(t \wedge \theta_{d}\right)\right) \\
& =V\left(X_{0}, r_{0}\right)+E \int_{0}^{t \wedge \theta_{d}} L V(X(s), X(q s), r(s)) d s .
\end{aligned}
$$


By (41), we have

$$
\begin{aligned}
& E V\left(X\left(t \wedge \theta_{d}\right), r\left(t \wedge \theta_{d}\right)\right) \\
& \leq V\left(X_{0}, r_{0}\right)+K E \int_{0}^{t \wedge \theta_{d}}\left[1-\lambda_{1} V(X(s), r(s))\right. \\
& \left.+\lambda_{2} V(X(q s), r(s))\right] d s \quad(46) \\
& \leq V\left(X_{0}, r_{0}\right)+K T \\
& +K\left(\lambda_{1}+\lambda_{2}\right) \int_{0}^{t \wedge \theta} \sup _{0 \leq u \leq s} E V(X(u), r(u)) d s .
\end{aligned}
$$

Thus, for any $t_{1} \in[0, T]$, it follows that

$$
\begin{array}{rl}
\sup _{0 \leq t \leq t_{1}} & E V\left(X\left(t \wedge \theta_{d}\right), r\left(t \wedge \theta_{d}\right)\right) \\
\leq & V\left(X_{0}, r_{0}\right)+K T \\
& \quad+\left(\lambda_{1}+\lambda_{2}\right) K \int_{0}^{t_{1}} \sup _{0 \leq t \leq s} E V\left(X\left(t \wedge \theta_{d}\right), r\left(t \wedge \theta_{d}\right)\right) d s .
\end{array}
$$

Using the Gronwall inequality, we obtain that

$$
\begin{array}{rl}
\sup _{0 \leq t \leq T} & E V\left(x\left(t \wedge \theta_{d}\right), r\left(t \wedge \theta_{d}\right)\right) \\
& \leq\left[V\left(X_{0}, r_{0}\right)+K T\right] e^{\left(\lambda_{1}+\lambda_{2}\right) K T} .
\end{array}
$$

Let $\mathscr{V}_{d}=\inf \{V(x, i):|x| \geq d\}$. By (40), we have $\lim _{d \rightarrow \infty} \mathscr{V}_{d}=\infty$. Noting that $\left|X\left(\theta_{d}\right)\right|=d$, as $\theta_{d}<T$, we derive from (48) that

$$
\begin{aligned}
& {\left[V\left(X_{0}, r_{0}\right)+K T\right] e^{\left(\lambda_{1}+\lambda_{2}\right) K T}} \\
& \quad \geq \sup _{0 \leq t \leq T} E V\left(x\left(t \wedge \theta_{d}\right), r\left(t \wedge \theta_{d}\right)\right) \\
& \quad \geq E\left[V\left(X\left(\theta_{d}\right), r\left(\theta_{d}\right)\right) I_{\left\{\theta_{d}<T\right\}}(w)\right] \\
& \quad \geq \mathscr{V}_{d} P\left(\theta_{d}<T\right) .
\end{aligned}
$$

That is,

$$
P\left(\theta_{d}<T\right) \leq \frac{\left[V\left(X_{0}, r_{0}\right)+K T\right] e^{\left(\lambda_{1}+\lambda_{2}\right) K T}}{\mathscr{V}_{d}} .
$$

Recall that $\mathscr{V}_{d} \rightarrow \infty$ as $d \rightarrow \infty$. For a given $T, X_{0}$, and $r(0)$, it follows that

$$
\frac{\left[V\left(X_{0}, r_{0}\right)+K T\right] e^{\left(\lambda_{1}+\lambda_{2}\right) K T}}{\mathscr{V}_{d}} \longrightarrow 0,
$$

as $d \rightarrow \infty$. Let

$$
\frac{\varepsilon}{3}=\frac{\left[V\left(X_{0}, r_{0}\right)+K T\right] e^{\left(\lambda_{1}+\lambda_{2}\right) K T}}{\mathscr{V}_{d}} \in(0,1) .
$$

Thus we have

$$
P\left(\theta_{d}<T\right) \leq \frac{\varepsilon}{3} .
$$

Step 2. We will give the estimate of $P\left(\rho_{d}<T\right)$. By (14), applying the Itô's formula to $V(Y(t), r(t))$ yields

$$
\begin{aligned}
& d V(Y(t), r(t)) \\
& =V_{x}(Y(t), r(t)) f\left(Z_{1}(t), Z_{2}(t), \bar{r}(t)\right) \\
& +\frac{1}{2} \operatorname{trace}\left[g^{\top}\left(Z_{1}(t), Z_{2}(t), \bar{r}(t)\right)\right. \\
& \left.\times V_{x x}(Y(t), r(t)) g\left(Z_{1}(t), Z_{2}(t), \bar{r}(t)\right)\right] d t \\
& +V_{x}(Y(t), r(t)) g\left(Z_{1}(t), Z_{2}(t), \bar{r}(t)\right) d W(t) \\
& +\int_{R^{n}}\left[V\left(Y(t)+h\left(Z_{1}(t), Z_{2}(t), \bar{r}(t), u\right), r(t)\right)\right. \\
& -V(Y(t), r(t))-V_{x}(Y(t), r(t)) \\
& \left.\times h\left(Z_{1}(t), Z_{2}(t), \bar{r}(t), u\right)\right] \Pi(d u) d t \\
& +\int_{R^{n}}\left[V\left(Y(t)+h\left(Z_{1}(t), Z_{2}(t), \bar{r}(t), u\right), r(t)\right)\right. \\
& -V(Y(t), r(t))] \widetilde{N}(d t, d u) \\
& +\sum_{j=1}^{N} \gamma_{r(t) j} V(Y(t), j) d t .
\end{aligned}
$$

By (7), we have

$$
\begin{aligned}
& d V(Y(t), r(t)) \\
& =L V\left(Z_{1}(t), Z_{2}(t), \bar{r}(t)\right) d t \\
& +\left[V_{x}(Y(t), r(t))-V_{x}\left(Z_{1}(t), \bar{r}(t)\right)\right] \\
& \times f\left(Z_{1}(t), Z_{2}(t), \bar{r}(t)\right) d t \\
& +\frac{1}{2} \operatorname{trace}\left[g^{\top}\left(Z_{1}(t), Z_{2}(t), \bar{r}(t)\right)\right. \\
& \quad \times\left[V_{x x}(Y(t), r(t))-V_{x x}\left(Z_{1}(\mathrm{t}), \bar{r}(t)\right)\right] \\
& \left.\quad \times g\left(Z_{1}(t), Z_{2}(t), \bar{r}(t)\right)\right] d t \\
& +\int_{R^{n}}\left\{\left[V\left(Y(t)+h\left(Z_{1}(t), Z_{2}(t), \bar{r}(t), u\right), r(t)\right)\right.\right. \\
& \left.\quad-V\left(Z_{1}(t)+h\left(Z_{1}(t), Z_{2}(t), \bar{r}(t), u\right), \bar{r}(t)\right)\right] \\
& \quad-\left[V(Y(t), r(t))-V\left(Z_{1}(t), \bar{r}(t)\right)\right] \\
& \quad-\left[V_{x}(Y(t), r(t))-V_{x}\left(Z_{1}(t), \bar{r}(t)\right)\right] \\
& \left.\times h\left(Z_{1}(t), Z_{2}(t), \bar{r}(t), u\right)\right\} \Pi(d u) d t \\
& +V_{x}(Y(t), r(t)) g\left(Z_{1}(t), Z_{2}(t), \bar{r}(t)\right) d W(t)
\end{aligned}
$$




$$
\begin{aligned}
& +\int_{R^{n}}\left[V\left(Y(t)+h\left(Z_{1}(t), Z_{2}(t), \bar{r}(t), u\right), r(t)\right)\right. \\
& -V(Y(t), r(t))] \widetilde{N}(d t, d u) \\
& +\sum_{j=1}^{N} \gamma_{r(t) j} V(Y(t), j) d t-\sum_{j=1}^{N} \gamma_{\bar{r}(t) j} V\left(Z_{1}(t), j\right) d t
\end{aligned}
$$

Integrating from 0 to $\rho_{d} \wedge t$, taking expectations, and by (41), we have

$$
\begin{aligned}
& E V\left(Y\left(t \wedge \rho_{d}\right), r\left(t \wedge \rho_{d}\right)\right) \\
& \leq V\left(X_{0}, r_{0}\right)+K T+\lambda_{1} K E \int_{0}^{\rho_{d} \wedge t} V(Y(s), r(s)) d s \\
& \quad+\lambda_{2} K E \int_{0}^{\rho_{d} \wedge t} V(Y(q s), r(s)) d s \\
& \quad-\lambda_{1} K E \int_{0}^{\rho_{d} \wedge t}\left[V\left(Z_{1}(s), \bar{r}(s)\right)+V(Y(s), r(s))\right] d s \\
& \quad+\mathscr{I}_{1}+\mathscr{I}_{2}+\mathscr{I}_{3}+\mathscr{I}_{4}+\mathscr{I}_{5},
\end{aligned}
$$

where

$$
\begin{aligned}
& \mathscr{I}_{1}=\lambda_{2} K E \int_{0}^{\rho_{d} \wedge t}\left|V\left(Z_{2}(s), \bar{r}(s)\right)-V(Y(q s), r(s))\right| d s, \\
& \mathscr{I}_{2}=E \int_{0}^{\rho_{d} \wedge t}\left|V_{x}(Y(s), r(s))-V_{x}\left(Z_{1}(s), \bar{r}(s)\right)\right| \\
& \times f\left(Z_{1}(s), Z_{2}(s), \bar{r}(s)\right) d s, \\
& \mathscr{I}_{3}=\frac{1}{2} E \int_{0}^{\rho_{d} \wedge t}\left|V_{x x}(Y(s), r(s))-V_{x x}\left(Z_{1}(s), \bar{r}(s)\right)\right| \\
& \times\left|g\left(Z_{1}(s), Z_{2}(s), \bar{r}(s)\right)\right|^{2} d s, \\
& \mathscr{I}_{4}=E \int_{0}^{\rho_{d} \wedge t} \int_{R^{n}}\left[\mid V\left(Y(s)+h\left(Z_{1}(s), Z_{2}(s)\right. \text {, }\right.\right. \\
& \bar{r}(s), u), r(s)) \\
& -V\left(Z_{1}(s)\right. \\
& \left.+h\left(Z_{1}(s), Z_{2}(s), \bar{r}(s), u\right), \bar{r}(s)\right) \mid \\
& +\left|V(Y(s), r(s))-V\left(Z_{1}(s), \bar{r}(s)\right)\right| \\
& +\left|V_{x}(Y(s), r(s))-V_{x}\left(Z_{1}(s), \bar{r}(s)\right)\right| \\
& \left.\times\left|h\left(Z_{1}(s), Z_{2}(s), \bar{r}(s), u\right)\right|\right] \pi(d u) d s, \\
& \mathscr{I}_{5}=E \int_{0}^{\rho_{d} \wedge t} \sum_{j=1}^{N}\left|\gamma_{r(s) j} V(Y(s), j)\right| d s \\
& -E \int_{0}^{\rho_{d} \wedge t} \sum_{j=1}^{N}\left|\gamma_{\bar{r}(s) j} V\left(Z_{1}(s), j\right)\right| d s .
\end{aligned}
$$

By (42) and Young's inequality, we have

$$
\begin{aligned}
\mathscr{I}_{1} \leq & \lambda_{2} K E \int_{0}^{\rho_{d} \wedge t}\left|V\left(Z_{2}(s), \bar{r}(s)\right)-V(Y(q s), \bar{r}(s))\right| d s \\
& +\lambda_{2} K E \int_{0}^{\rho_{d} \wedge t}|V(Y(q s), \bar{r}(s))-V(Y(q s), r(s))| d s \\
\leq & \lambda_{2} K L_{d} E \int_{0}^{\rho_{d} \wedge t}\left|Z_{2}(s)-Y(q s)\right| d s \\
& +\lambda_{2} K E \int_{0}^{T}|V(Y(q s), \bar{r}(s))-V(Y(q s), r(s))| d s \\
\leq & \lambda_{2} K L_{d} \int_{0}^{\rho_{d} \wedge t}\left(E\left|Z_{2}(s)-Y(q s)\right|^{2}\right)^{1 / 2} d s+\lambda_{2} \mathscr{J} .
\end{aligned}
$$

Let $N=[T / h]$, the integer part of $T / h$, and let $I_{G}$ be the indicator function of the set $G$. Then

$$
\begin{aligned}
\mathscr{J} \leq & K \sum_{n=0}^{N} E \int_{n h}^{(n+1) h}\left|V\left(Y_{[q n]}, r_{n}^{h}\right)-V\left(Y_{[q n]}, r(s)\right)\right| d s \\
\leq & K \sum_{n=0}^{N} E \int_{n h}^{(n+1) h}\left|V\left(Y_{[q n]}, r_{n}^{h}\right)\right| \\
& +\left|f\left(Y_{[q n]}, r(s)\right)\right| I_{\left\{r(s) \neq r_{n}^{h}\right\}} d s .
\end{aligned}
$$

By setting $V_{1}=\sup _{|x| \leq R, i \in S} V(x, i)$ and using the Markov property, we have

$$
\begin{aligned}
\mathscr{J} \leq & 2 K V_{1} \sum_{n=0}^{N} E \int_{n h}^{(n+1) h} I_{\left\{r(s) \neq r_{n}^{h}\right\}} d s \\
= & 2 K V_{1} \sum_{n=0}^{N} \int_{n h}^{(n+1) h} E\left[E\left[I_{\left\{r(s) \neq r_{n}^{h}\right\}}\right] \mid r_{n}^{h}\right] d s \\
= & 2 K V_{1} \sum_{n=0}^{N} \int_{n h}^{(n+1) h} E\left[\sum_{i \in S} I_{\left\{r_{n}^{h}=i\right\}} P\left(r(s) \neq i \mid r_{n}^{h}=i\right)\right] d s \\
= & 2 K V_{1} \sum_{n=0}^{N} \int_{n h}^{(n+1) h}\left[\sum_{i \in S} I_{\left\{r_{n}^{h}=i\right\}} \sum_{j \neq i}\left(\gamma_{i j}(s-n h)+\circ(s-n h)\right)\right] \\
\leq & 2 K V_{1} \sum_{n=0}^{N} \int_{n h}^{(n+1) h} E\left[\left(\max _{1 \leq i \leq N}\left(-\gamma_{i i}\right) h+\circ(h)\right) \sum_{i \in S} I_{\left\{r_{n}^{h}=i\right\}}\right] d s \\
= & 2 K V_{1}\left[\max _{1 \leq i \leq N}\left(-\gamma_{i i}\right) h+\circ(h)\right] T . \\
& \times d s
\end{aligned}
$$


Inserting (60) into (58), we have, by Lemma 5,

$$
\begin{aligned}
\mathscr{I}_{1} \leq & \lambda_{2} K L_{d} \int_{0}^{\rho_{d} \wedge t}\left(E\left|Z_{2}(s)-Y(q s)\right|^{2}\right)^{1 / 2} d s \\
& +2 \lambda_{2} K V_{1} \max _{1 \leq i \leq N}\left(-\gamma_{i i}\right) h+\circ(h) T \\
\leq & \lambda_{2} K L_{d} \sqrt{C_{2}(d) T h^{1 / 2}+2 \lambda_{2} K V_{1} T \max _{1 \leq i \leq N}\left(-\gamma_{i i}\right)[h+\circ(h)] .}
\end{aligned}
$$

Similarly, by Assumptions 2, 3, and 11, Lemma 5, and Markov property, we have

$$
\begin{aligned}
\mathscr{I}_{2} \leq & E \int_{0}^{\rho_{d} \wedge t}\left|V_{x}(Y(s), r(s))-V_{x}\left(Z_{1}(s), r(s)\right)\right| \\
& \times\left|f\left(Z_{1}(s), Z_{2}(s), \bar{r}(s)\right)\right| d s \\
& +E \int_{0}^{\rho_{d} \wedge t}\left|V_{x}\left(Z_{1}(s), r(s)\right)-V_{x}\left(Z_{1}(s), \bar{r}(s)\right)\right| \\
& \times\left|f\left(Z_{1}(s), Z_{2}(s), \bar{r}(s)\right)\right| d s \\
\leq & 2 \sqrt{K_{d}} d E \int_{0}^{\rho_{d} \wedge t}\left|V_{x}(Y(s), r(s))-V_{x}\left(Z_{1}(s), r(s)\right)\right| d s \\
& +2 \sqrt{K_{d}} d E \int_{0}^{\rho_{d} \wedge t} \mid V_{x}\left(Z_{1}(s), r(s)\right) \\
\leq & 2 \sqrt{K_{d}} d L_{d} E \int_{0}^{\rho_{d} \wedge t}\left|Y(s)-Z_{1}(s)\right| d s \\
& +2 d \sqrt{K_{d}} E \int_{0}^{T}\left|V_{x}\left(Z_{1}(s), r(s)\right)-V_{x}\left(Z_{1}(s), \bar{r}(s)\right)\right| d s \\
\leq & \left.2 d \sqrt{K_{d}} L_{d} \sqrt{C_{1}(d) T h^{1 / 2}}(s), \bar{r}(s)\right) \mid d s \\
& +4 \sqrt{K_{d}} d V_{2} T \max _{1 \leq i \leq N}\left(-\gamma_{i i}\right)[h+\circ(h)]
\end{aligned}
$$

where $V_{2}=\sup _{|x| \leq R, i \in S} V_{x}(x, i)$. For the term $\mathscr{I}_{3}$ in (56), by Assumptions 2, 3, and 11 and Lemma 5, we have

$$
\begin{aligned}
\mathscr{I}_{3} \leq \frac{1}{2} E \int_{0}^{\rho_{d} \wedge t} & \left|V_{x x}(Y(s), r(s))-V_{x x}\left(Z_{1}(s), r(s)\right)\right| \\
& \times\left|g\left(Z_{1}(s), Z_{2}(s), \bar{r}(s)\right)\right|^{2} d s \\
+ & \frac{1}{2} E \int_{0}^{\rho_{d} \wedge t}\left|V_{x x}\left(Z_{1}(s), r(s)\right)-V_{x x}\left(Z_{1}(s), \bar{r}(s)\right)\right| \\
& \times\left|g\left(Z_{1}(s), Z_{2}(s), \bar{r}(s)\right)\right|^{2} d s
\end{aligned}
$$

$$
\begin{aligned}
& \leq 2 d^{2} K_{d} E \int_{0}^{\rho_{d} \wedge t}\left|V_{x x}(Y(s), r(s))-V_{x x}\left(Z_{1}(s), r(s)\right)\right| d s \\
& +2 d^{2} K_{d} E \int_{0}^{\rho_{d} \wedge t} \mid V_{x x}\left(Z_{1}(s), r(s)\right) \\
& \quad-V_{x x}\left(Z_{1}(s), \bar{r}(s)\right) \mid d s \\
& \leq 2 d^{2} K_{d} L_{d} E \int_{0}^{\rho_{d} \wedge t}\left|Y(s)-Z_{1}(s)\right| d s \\
& +2 d^{2} K_{d} E \int_{0}^{T} \mid V_{x x}\left(Z_{1}(s), r(s)\right) \\
& \quad-V_{x x}\left(Z_{1}(s), \bar{r}(s)\right) \mid d s \\
& \leq \quad 2 d^{2} K_{d} L_{d} \sqrt{C_{1}(d) T h^{1 / 2}} \\
& +4 d^{2} K_{d} V_{3} T \max _{1 \leq i \leq \mathrm{N}}\left(-\gamma_{i i}\right)[h+\circ(h)],
\end{aligned}
$$

where $V_{3}=\sup _{|x| \leq R, i \in S} V_{x x}(x, i)$. For the term $\mathscr{I}_{4}$ in (56), by Assumptions 2, 3, and 11 and Lemma 5, we have

$$
\begin{aligned}
& \mathscr{I}_{4} \leq E \int_{0}^{\rho_{d} \wedge t} \int_{R^{n}} \mid V(Y(s) \\
& \left.+h\left(Z_{1}(s), Z_{2}(s), \bar{r}(s), u\right), r(s)\right) \\
& -V\left(Z_{1}(s)+h\left(Z_{1}(s), Z_{2}(s), \bar{r}(s), u\right),\right. \\
& r(s)) \mid \pi(d u) d s \\
& +E \int_{0}^{\rho_{d} \wedge t} \int_{R^{n}} \mid V\left(Z_{1}(s)\right. \\
& \left.+h\left(Z_{1}(s), Z_{2}(s), \bar{r}(s), u\right), r(s)\right) \\
& -V\left(Z_{1}(s)\right. \\
& \left.+h\left(Z_{1}(s), Z_{2}(s), \bar{r}(s), u\right), \bar{r}(s)\right) \mid \\
& \times \pi(d u) d s \\
& +E \int_{0}^{\rho_{d} \wedge t} \int_{R^{n}}\left|V(Y(s), r(s))-V\left(Z_{1}(s), r(s)\right)\right| \\
& \times \pi(d u) d s \\
& +E \int_{0}^{\rho_{d} \wedge t} \int_{R^{n}}\left|V\left(Z_{1}(s), r(s)\right)-V\left(Z_{1}(s), \bar{r}(s)\right)\right| \\
& \times \pi(d u) d s \\
& +E \int_{0}^{\rho_{d} \wedge t} \int_{R^{n}}\left|V_{x}(Y(s), r(s))-V_{x}\left(Z_{1}(s), r(s)\right)\right| \\
& \times\left|h\left(Z_{1}(s), Z_{2}(s), \bar{r}(s), u\right)\right| \pi(d u) d s \\
& +E \int_{0}^{\rho_{d} \wedge \mathrm{t}} \int_{R^{n}}\left|V_{x}\left(Z_{1}(s), r(s)\right)-V_{x}\left(Z_{1}(s), \bar{r}(s)\right)\right| \\
& \times\left|h\left(Z_{1}(s), Z_{2}(s), \bar{r}(s), u\right)\right| \pi(d u) d s
\end{aligned}
$$




$$
\begin{aligned}
& \leq 2 L_{d} E \int_{0}^{\rho_{d} \wedge t} \int_{R^{n}}\left|Y(s)-Z_{1}(s)\right| \pi(d u) d s \\
& +2 d \sqrt{K_{d} \int_{R^{n}} \pi(d u)} \\
& \times E \int_{0}^{\rho_{d} \wedge t}\left|V_{x}(Y(s), r(s))-V_{x}\left(Z_{1}(s), r(s)\right)\right| d s \\
& +\int_{R^{n}} \pi(d u) E \\
& \times \int_{0}^{\rho_{d} \wedge t}\left|V\left(Z_{1}(s), r(s)\right)-V\left(Z_{1}(s), \bar{r}(s)\right)\right| d s \\
& +2 d \sqrt{K_{d} \int_{R^{n}} \pi(d u)} \\
& \times E \int_{0}^{\rho_{d} \wedge t}\left|V_{x}\left(Z_{1}(s), r(s)\right)-V_{x}\left(Z_{1}(s), \bar{r}(s)\right)\right| d s \\
& +E \int_{0}^{\rho_{d} \wedge t} \int_{R^{n}} \mid V\left(Z_{1}(s)\right. \\
& \left.+h\left(Z_{1}(s), Z_{2}(s), \bar{r}(s), u\right), r(s)\right) \\
& -V\left(Z_{1}(s)\right. \\
& \left.+h\left(Z_{1}(s), Z_{2}(s), \bar{r}(s), u\right), \bar{r}(s)\right) \mid \\
& \times \pi(d u) d s \\
& \leq 2\left[\int_{R^{n}} \pi(d u)+2 d \sqrt{K_{d} \int_{R^{n}} \pi(d u)}\right] \\
& \times \sqrt{C_{1}(d)} L_{d} T h^{1 / 2} \\
& +2\left[\int_{R^{n}} \pi(d u) V_{1}+2 d \sqrt{K_{d} \int_{R^{n}} \pi(d u)} V_{2}\right] \\
& \times T \max _{1 \leq i \leq N}\left(-\gamma_{i i}\right)[h+\circ(h)]+\mathscr{L},
\end{aligned}
$$

where

$$
\begin{gathered}
\mathscr{L} \leq E \int_{0}^{\rho_{d} \wedge t} \int_{R^{n}} \mid V\left(Z_{1}(s)+h\left(Z_{1}(s), Z_{2}(s), \bar{r}(s), u\right), r(s)\right) \\
-V\left(Z_{1}(s), \bar{r}(s)\right) \mid \pi(d u) d s \\
+E \int_{0}^{\rho_{d} \wedge t} \int_{R^{n}} \mid V\left(Z_{1}(s), r(s)\right) \\
-V\left(Z_{1}(s), \bar{r}(s)\right) \mid \pi(d u) d s \\
+E \int_{0}^{\rho_{d} \wedge t} \int_{R^{n}} \mid V\left(Z_{1}(s), \bar{r}(s)\right) \\
-V\left(Z_{1}(s)+h\left(Z_{1}(s), Z_{2}(s), \bar{r}(s), u\right),\right. \\
\bar{r}(s)) \mid \pi(d u) d s
\end{gathered}
$$

$$
\begin{aligned}
& \leq 4 d L_{d} \sqrt{K_{d} \int_{R^{n}} \pi(d u) T} \\
& \quad+2 V_{1} T \int_{R^{n}} \pi(d u) \max _{1 \leq i \leq N}\left(-\gamma_{i i}\right)[h+\circ(h)] .
\end{aligned}
$$

Inserting (65) into (64), we have

$$
\begin{aligned}
\mathscr{I}_{4} \leq & 2\left[\int_{R^{n}} \pi(d u)+2 d \sqrt{K_{d} \int_{R^{n}} \pi(d u)}\right] \sqrt{C_{1}(d)} L_{d} T h^{1 / 2} \\
& +4 d L_{d} \sqrt{K_{d} \int_{R^{n}} \pi(d u) T} \\
& +4\left[\int_{R^{n}} \pi(d u) V_{1}+d \sqrt{K_{d} \int_{R^{n}} \pi(d u) V_{2}}\right] \\
& \times T \max _{1 \leq i \leq N}\left(-\gamma_{i i}\right)[h+\circ(h)] .
\end{aligned}
$$

For the term $\mathscr{I}_{5}$, by Assumption 11, equation (56), and Lemma 5, we have

$$
\begin{aligned}
\mathscr{I}_{5} \leq & E \int_{0}^{\rho_{d} \wedge t} \sum_{j=1}^{N}\left|\gamma_{r(s) j}\right|\left|V(Y(s), j)-V\left(Z_{1}(s), j\right)\right| d s \\
& +E \int_{0}^{\rho_{d} \wedge t} \sum_{j=1}^{N}\left|\gamma_{r(s) j}-\gamma_{\bar{r}(s) j}\right|\left|V\left(Z_{1}(s), j\right)\right| d s \\
\leq & N \max _{1 \leq i \leq N}\left(-\gamma_{i i}\right) L_{d} \sqrt{C_{1}(d) T h^{1 / 2}+2 V_{1} N T \max _{1 \leq i \leq N}\left(-\gamma_{i i}\right) .}
\end{aligned}
$$

Combing these inequalities and (53), we derive that, for $t_{1} \epsilon$ $[0, T]$,

$$
\begin{array}{rl}
\sup _{0 \leq t \leq t_{1}} & E V\left(Y\left(t \wedge \rho_{d}\right), r\left(t \wedge \rho_{d}\right)\right) \\
\leq & V\left(X_{0}, r_{0}\right) \\
& +\left[K+4 d L_{d} \sqrt{K_{d} \int_{R^{n}} \pi(d u)}+2 V_{1} N \max _{1 \leq i \leq N}\left(-\gamma_{i i}\right)\right] T \\
& +\left(\lambda_{1}+\lambda_{2}\right) K \\
& \times \int_{0}^{t_{1}} \sup _{0 \leq t \leq s} E V\left(Y\left(t \wedge \rho_{d}\right)\right) d s+M_{1} h^{1 / 2}+M_{2}[h+\circ(h)],
\end{array}
$$


where

$$
\begin{aligned}
M_{1}= & L_{d} \sqrt{C_{1}(d) T}\left[2 d \sqrt{K_{d}}+2 d^{2} K_{d}+2 \int_{R^{n}} \pi(d u)\right. \\
& \left.+4 d \sqrt{K_{d} \int_{R^{n}} \pi(d u)}+N \max _{1 \leq i \leq N}\left(-\gamma_{i i}\right)\right] \\
& +\lambda_{2} K L_{d} \sqrt{C_{2}(d) T}, \\
M_{2}= & \left(2 \lambda_{2} K+4 \int_{R^{n}} \pi(d u)\right) V_{1} \\
& +\left(4 d \sqrt{K_{d}}+4 d \sqrt{K_{d} \int_{R^{n}} \pi(d u)}\right) V_{2} \\
& \left.+4 d^{2} K_{d} V_{3}\right] \max \left(-\gamma_{i i}\right) .
\end{aligned}
$$

For arbitrary $0 \leq t_{1} \leq T$, by the Gronwall inequality, we get

$$
\begin{array}{rl}
\sup _{0 \leq t \leq T} & E V\left(Y\left(t \wedge \rho_{d}\right), r\left(t \wedge \rho_{d}\right)\right) \\
\leq & \left\{V\left(X_{0}, r_{0}\right)\right. \\
+ & {\left[K+4 d L_{d} \sqrt{K_{d} \int_{R^{n}} \pi(d u)}+2 V_{1} N \max _{1 \leq i \leq N}\left(-\gamma_{i i}\right)\right] T} \\
+ & \left.M_{1} h^{1 / 2}+M_{2}[h+\circ(h)]\right\} e^{\left(\lambda_{1}+\lambda_{2}\right) K T} .
\end{array}
$$

Noting that $\left|Y\left(\rho_{d}\right)\right|=d$, as $\rho_{d}<T$, we derive from (70) that

$$
\begin{aligned}
& \left\{V\left(X_{0}, r_{0}\right)\right. \\
& +\left[K+4 d L_{d} \sqrt{K_{d} \int_{R^{n}} \pi(d u)}+2 V_{1} N \max _{1 \leq i \leq N}\left(-\gamma_{i i}\right)\right] T \\
& \left.+M_{1} h^{1 / 2}+M_{2}[h+\circ(h)]\right\} e^{\left(\lambda_{1}+\lambda_{2}\right) K T} \\
& \quad \geq E V\left(Y\left(t \wedge \rho_{d}\right), r\left(t \wedge \rho_{d}\right)\right) \\
& \quad \geq E\left[V\left(Y\left(\rho_{d}\right), r\left(\rho_{d}\right)\right) I_{\left\{\rho_{d}<T\right\}}(w)\right] \\
& \quad \geq \mathscr{V}_{d} P\left(\rho_{d}<T\right) .
\end{aligned}
$$

$$
\text { So we have }
$$

$$
\begin{aligned}
P\left(\rho_{d}<T\right) & \\
\leq & \frac{\left\{V\left(X_{0}, r_{0}\right)+\left[K+4 d L_{d} \sqrt{K_{d} \int_{R^{n}} \pi(d u)}\right] T\right\} e^{\left(\lambda_{1}+\lambda_{2}\right) K T}}{\mathscr{V}_{d}} \\
& +\frac{\left\{\left[2 V_{1} N \max _{1 \leq i \leq N}\left(-\gamma_{i i}\right)\right] T\right\} e^{\left(\lambda_{1}+\lambda_{2}\right) K T}}{\mathscr{V}_{d}} \\
& +\frac{\left\{M_{1} h^{1 / 2}+M_{2}[h+\circ(h)]\right\} e^{\left(\lambda_{1}+\lambda_{2}\right) K T}}{\mathscr{V}_{d}}
\end{aligned}
$$

Step 3. Let $\epsilon, \delta \in(0,1)$ be arbitrarily small. By setting

$$
\bar{\Omega}=\left\{w: \sup _{0 \leq t \leq T}|Y(t)-X(t)|^{2} \geq \delta\right\}
$$

and using Theorem 4, we have

$$
\begin{aligned}
C_{d} & {[h+\circ(h)] } \\
& \geq E\left[\sup _{0 \leq t \leq T}|Y(t)-X(t)|^{2} I_{\tau_{d} \geq T}(w)\right] \\
& \geq E\left[\sup _{0 \leq t \leq T}|Y(t)-X(t)|^{2} I_{\tau_{d} \geq T}(w) I_{\bar{\Omega}}(w)\right] \\
& \geq \delta E\left[I_{\tau_{d} \geq T}(w) I_{\bar{\Omega}}(w)\right]=\delta P\left(\left\{\tau_{d} \geq T\right\} \cap \bar{\Omega}\right) \\
& \geq \delta\left[P(\bar{\Omega})-P\left(\tau_{d}<T\right)\right] .
\end{aligned}
$$

Combing (53) and (72) together, one gets

$$
\begin{aligned}
P\left(\tau_{d}<T\right) & \leq\left(\rho_{d}<T\right)+P\left(\theta_{d}<T\right) \\
\leq & \frac{\varepsilon}{3} \\
& +\frac{\left\{V\left(X_{0}, r_{0}\right)+\left[K+4 d L_{d} \sqrt{K_{d} \int_{R^{n}} \pi(d u)}\right] T\right\} e^{\left(\lambda_{1}+\lambda_{2}\right) K T}}{\mathscr{V}_{d}} \\
& +\frac{\left\{\left[2 V_{1} N \max _{1 \leq i \leq N}\left(-\gamma_{i i}\right)\right] T\right\} e^{\left(\lambda_{1}+\lambda_{2}\right) K T}}{\mathscr{V}_{d}} \\
& +\frac{\left\{M_{1} h^{1 / 2}+M_{2}[h+\circ(h)]\right\} e^{\left(\lambda_{1}+\lambda_{2}\right) K T}}{\mathscr{V}_{d}} .
\end{aligned}
$$


Hence on using (74), we conclude that

$$
\begin{aligned}
P(\bar{\Omega}) & P\left(\sup _{0 \leq t \leq T}|Y(t)-X(t)|^{2} \geq \delta\right) \\
\leq & \frac{\left\{V\left(X_{0}, r_{0}\right)+\left[K+4 d L_{d} \sqrt{K_{d} \int_{R^{n}} \pi(d u)}\right] T\right\} e^{\left(\lambda_{1}+\lambda_{2}\right) K T}}{\mathscr{V}_{d}} \\
& +\frac{\left\{\left[2 V_{1} N \max _{1 \leq i \leq N}\left(-\gamma_{i i}\right)\right] T\right\} e^{\left(\lambda_{1}+\lambda_{2}\right) K T}}{\mathscr{V}_{d}} \\
& +\frac{\left\{M_{1} h^{1 / 2}+M_{2}[h+\circ(h)]\right\} e^{\left(\lambda_{1}+\lambda_{2}\right) K T}}{\mathscr{V}_{d}} \\
& +\frac{C_{d}[h+\circ(h)]+\frac{\varepsilon}{3} .}{\delta}
\end{aligned}
$$

Recalling that $\mathscr{V}_{d} \rightarrow \infty$ as $d \rightarrow \infty$, we can choose $d$ sufficiently large for

$$
\begin{aligned}
& \frac{\left\{V\left(X_{0}, r_{0}\right)+\left[K+4 d L_{d} \sqrt{K_{d} \int_{R^{n}} \pi(d u)}\right] T\right\} e^{\left(\lambda_{1}+\lambda_{2}\right) K T}}{\mathscr{V}_{d}} \\
& +\frac{\left\{\left[2 V_{1} N \max _{1 \leq i \leq N}\left(-\gamma_{i i}\right)\right] T\right\} e^{\left(\lambda_{1}+\lambda_{2}\right) K T}}{\mathscr{V}_{d}}<\frac{\varepsilon}{3}
\end{aligned}
$$

and then choose $h$ sufficiently small for

$$
\frac{\left\{M_{1} h^{1 / 2}+M_{2}[h+\circ(h)]\right\} e^{\left(\lambda_{1}+\lambda_{2}\right) K T}}{\mathscr{V}_{d}}+\frac{C_{d}}{\delta}[h+\circ(h)]<\frac{\varepsilon}{3}
$$

to obtain

$$
P\left(\sup _{0 \leq t \leq T}|Y(t)-X(t)|^{2} \geq \delta\right)<\varepsilon .
$$

The proof of Theorem 12 is now complete.

\section{An Example}

In this section, we construct one example to demonstrate the effectiveness of this theory. Let $r(t)$ be a right-continuous Markov chain taking values in $S=\{1,2\}$ with the generator

$$
\Gamma=\left(\gamma_{i j}\right)_{2 \times 2}=\left[\begin{array}{cc}
-2 & 2 \\
2 & -2
\end{array}\right]
$$

Let $\widetilde{N}(d t, d u)$ be a compensated Poisson random measures and is given by $\pi(d u) d t=\lambda f(u) d u d t$, where $\lambda=2$ and

$$
f(u)=\frac{1}{\sqrt{2 \pi u}} e^{-(\operatorname{lnu})^{2} / 2}, \quad 0 \leq u<\infty
$$

is the density function of a lognormal random variable. Of course $\widetilde{N}(d t, d u)$ and $r(t)$ are assumed to be independent. Consider a linear stochastic pantograph delay equations with Markovian switching and pure jumps

$$
\begin{aligned}
d X(t)= & a(r(t)) X(t) d t \\
& +\int_{|u| \leq 1} u b(r(t)) X(0.5 t) \widetilde{N}(d t, d u) .
\end{aligned}
$$

Here $a(1)=-3, a(2)=-1, b(1)=1 / \sqrt{10}$, and $b(2)=1 / \sqrt{6}$. Then (82) can be regarded as the result of the two equations

$$
\begin{gathered}
d X(t)=-3 X(t) d t+\frac{1}{\sqrt{10}} \int_{|u| \leq 1} u X(0.5 t) \widetilde{N}(d t, d u), \\
d X(t)=-X(t) d t+\frac{1}{\sqrt{6}} \int_{|u| \leq 1} u X(0.5 t) \widetilde{N}(d t, d u),
\end{gathered}
$$

switching among each other according to the movement of the Markov chain $r(t)$. Obviously, (82) satisfies Assumptions 2 and 3. Given the stepsize $h$, we can have the Euler method

$$
\begin{aligned}
Y_{n+1}= & Y_{n}+a\left(r_{n}^{h}\right) Y_{n} h \\
& +\int_{t_{n}}^{t_{n+1}} \int_{|u| \leq 1} u b\left(r_{n}^{h}\right) Y_{[0.5 n]} \widetilde{N}(d t, d u),
\end{aligned}
$$

with $Y_{0}=X(0)$. Let $\bar{Z}_{1}(t)=Y_{n}, \bar{Z}_{2}(t)=Y_{[0.5 n]}$, and $\bar{r}(t)=$ $r_{n}^{h}$, for $t \in\left[t_{n}, t_{n+1}\right)$. Then we define the continuous Euler approximate solution

$$
\begin{aligned}
Y(t)= & Y(0)+\int_{0}^{t} a(\bar{r}(s)) \bar{Z}_{1}(s) d s \\
& +\int_{0}^{t} \int_{|u| \leq 1} u b(\bar{r}(s)) \bar{Z}_{2}(s) \widetilde{N}(d s, d u) .
\end{aligned}
$$

Since the conditions of Theorem 4 are satisfied, then the approximate solution (85) will converge to the true solution of (82) in the sense of the $L^{2}$-norm. To examine the convergence in the sense of the probability, we construct a function

$$
V(x, i)= \begin{cases}\beta_{1} x^{2}, & \text { if } i=1, \\ \beta_{2} x^{2}, & \text { if } i=2 .\end{cases}
$$

Then

$$
\begin{aligned}
& L V(x, y, i) \\
& = \begin{cases}\left(2 \beta_{2}-8 \beta_{1}\right) x^{2}+\frac{1}{10} \int_{|u| \leq 1} u^{2} \pi(d u) \beta_{1} y^{2}, & \text { if } i=1, \\
\left(2 \beta_{1}-4 \beta_{2}\right) x^{2}+\frac{1}{6} \int_{|u| \leq 1} u^{2} \pi(d u) \beta_{2} y^{2}, & \text { if } i=2 .\end{cases}
\end{aligned}
$$

From the properties of the lognormal distribution, we have

$$
\begin{aligned}
& L V(x, y, 1) \leq-\left(8-\frac{2 \beta_{2}}{\beta_{1}}\right) \beta_{1} x^{2}+\frac{1}{5} e^{2} \beta_{1} y^{2}, \\
& L V(x, y, 2) \leq-\left(4-\frac{2 \beta_{1}}{\beta_{2}}\right) \beta_{2} x^{2}+\frac{1}{3} e^{2} \beta_{2} y^{2} .
\end{aligned}
$$


If $6 /\left(12-e^{2}\right)<\left(\beta_{2} / \beta_{1}\right)<4-(1 / 10) e^{2}$, then it follows that Assumptions 10 and 11 are satisfied. Consequently, the approximate solution (85) will converge to the true solution of (82) for any $t \in[0, T]$ in the sense of Theorem 12 .

\section{Acknowledgment}

This work is sponsored by Qing Lan project of Jiangsu Province (2012) and supported by the Grant of Jiangsu Second Normal University (Jsie2011zd04) and the Jiangsu Government Overseas Study Scholarship.

\section{References}

[1] G. N. Milstein, Numerical Integration of Stochastic Differential Equations, vol. 313 of Mathematics and Its Applications, Kluwer Academic Publishers Group, Dordrecht, The Netherlands, 1995.

[2] C. T. H. Baker and E. Buckwar, "Numerical analysis of explicit one-step methods for stochastic delay differential equations," LMS Journal of Computation and Mathematics, vol. 3, pp. 315$335,2000$.

[3] X. Mao, Stochastic Differential Equations and Applications, Horwood, Chichester, UK, 1997.

[4] X. Mao, "Numerical solutions of stochastic differential delay equations under the generalized Khasminskii-type conditions," Applied Mathematics and Computation, vol. 217, no. 12, pp. 55125524, 2011.

[5] X. Mao, "Numerical solutions of stochastic functional differential equations," LMS Journal of Computation and Mathematics, vol. 6, pp. 141-161, 2003.

[6] X. Mao and S. Sabanis, "Numerical solutions of stochastic differential delay equations under local Lipschitz condition," Journal of Computational and Applied Mathematics, vol. 151, no. 1, pp. 215-227, 2003.

[7] F. Wu, X. Mao, and L. Szpruch, "Almost sure exponential stability of numerical solutions for stochastic delay differential equations," Numerische Mathematik, vol. 115, no. 4, pp. 681-697, 2010.

[8] F. Wu, X. Mao, and P. E. Kloeden, "Almost sure exponential stability of the Euler-Maruyama approximations for stochastic functional differential equations," Random Operators and Stochastic Equations, vol. 19, no. 2, pp. 165-186, 2011.

[9] E. Buckwar, "Introduction to the numerical analysis of stochastic delay differential equations," Journal of Computational and Applied Mathematics, vol. 125, no. 1-2, pp. 297-307, 2000.

[10] E. Buckwar, "One-step approximations for stochastic functional differential equations," Applied Numerical Mathematics, vol. 56, no. 5, pp. 667-681, 2006.

[11] U. Küchler and E. Platen, "Strong discrete time approximation of stochastic differential equations with time delay," Mathematics and Computers in Simulation, vol. 54, no. 1-3, pp. 189-205, 2000.

[12] Y. Hu, S. E. A. Mohammed, and F. Yan, "Discrete-time approximations of stochastic delay equations: the Milstein scheme," The Annals of Probability, vol. 32, no. 1A, pp. 265-314, 2004.

[13] C. T. H. Baker and E. Buckwar, "Continuous $\Theta$-methods for the stochastic pantograph equation," Electronic Transactions on Numerical Analysis, vol. 11, pp. 131-151, 2000.

[14] Z. Fan, M. Liu, and W. Cao, "Existence and uniqueness of the solutions and convergence of semi-implicit Euler methods for stochastic pantograph equations," Journal of Mathematical Analysis and Applications, vol. 325, no. 2, pp. 1142-1159, 2007.

[15] Y. Xiao and H. Zhang, "Convergence and stability of numerical methods with variable step size for stochastic pantograph differential equations," International Journal of Computer Mathematics, vol. 88, no. 14, pp. 2955-2968, 2011.

[16] M. Milošević and M. Jovanović, "A Taylor polynomial approach in approximations of solution to pantograph stochastic differential equations with Markovian switching," Mathematical and Computer Modelling, vol. 53, no. 1-2, pp. 280-293, 2011.

[17] L.-s. Wang, C. Mei, and H. Xue, "The semi-implicit Euler method for stochastic differential delay equations with jumps," Applied Mathematics and Computation, vol. 192, no. 2, pp. 567578, 2007.

[18] M. Wei, "Convergence of numerical solutions for variable delay differential equations driven by Poisson random jump measure," Applied Mathematics and Computation, vol. 212, no. 2, pp. 409-417, 2009.

[19] N. Jacob, Y. Wang, and C. Yuan, "Numerical solutions of stochastic differential delay equations with jumps," Stochastic Analysis and Applications, vol. 27, no. 4, pp. 825-853, 2009.

[20] F. Jiang, Y. Shen, and L. Liu, "Taylor approximation of the solutions of stochastic differential delay equations with Poisson jump," Communications in Nonlinear Science and Numerical Simulation, vol. 16, no. 2, pp. 798-804, 2011.

[21] G. Marion, X. Mao, and E. Renshaw, "Convergence of the Euler scheme for a class of stochastic differential equation," International Mathematical Journal, vol. 1, no. 1, pp. 9-22, 2002.

[22] N. Ikeda and S. Watanabe, Stochastic Differential Equations and Diffusion Processes, vol. 24 of North-Holland Mathematical Library, North-Holland, Amsterdam, The Netherlands, 2nd edition, 1989.

[23] W. J. Anderson, Continuous-Time Markov Chains, Springer Series in Statistics: Probability and Its Applications, Springer, New York, NY, USA, 1991.

[24] X. Mao and C. Yuan, Stochastic Differential Equations with Markovian Switching, Imperial College Press, London, UK, 2006. 


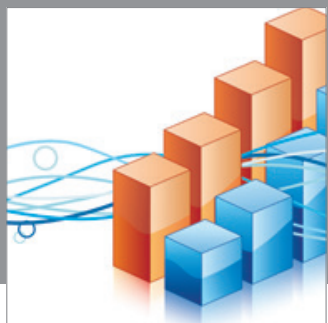

Advances in

Operations Research

mansans

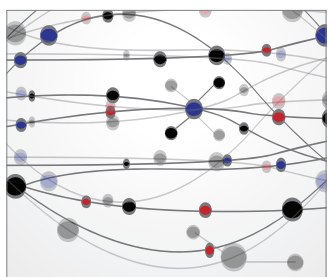

The Scientific World Journal
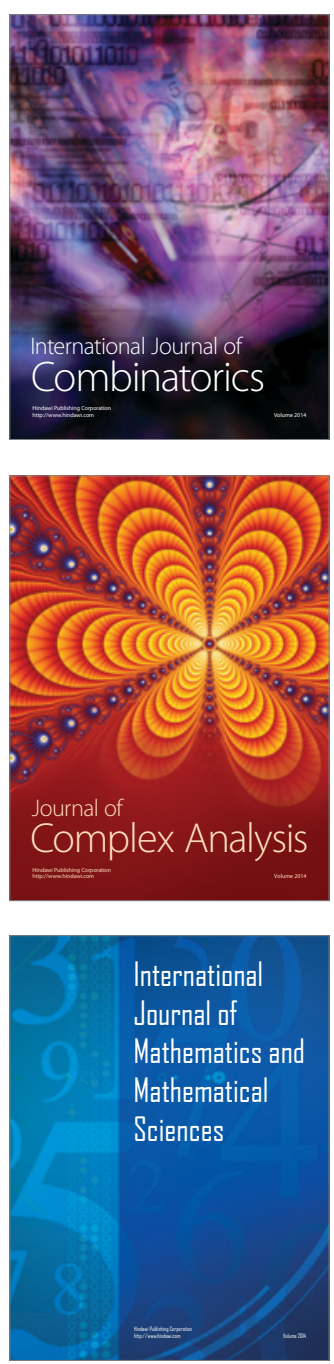
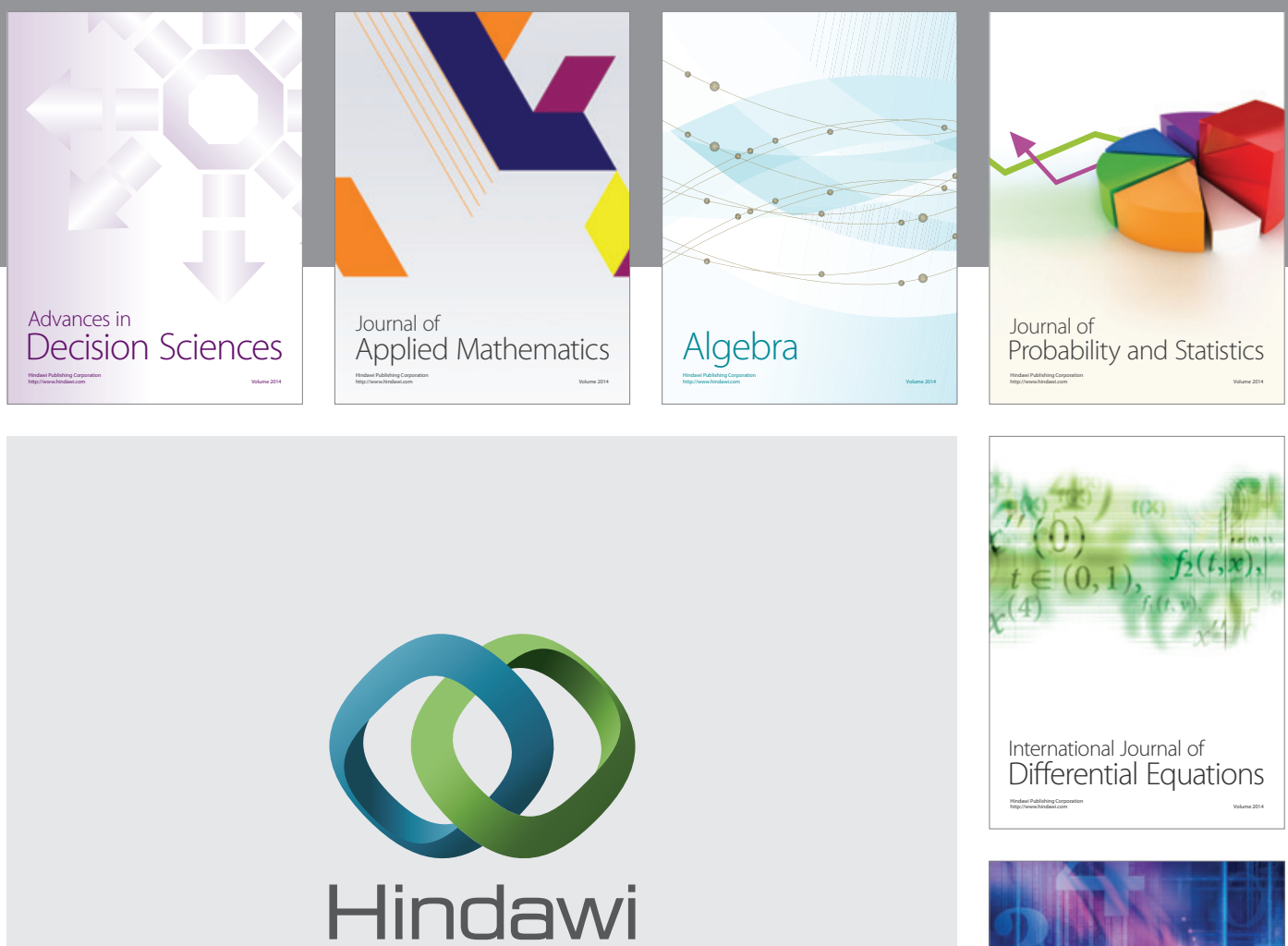

Submit your manuscripts at http://www.hindawi.com
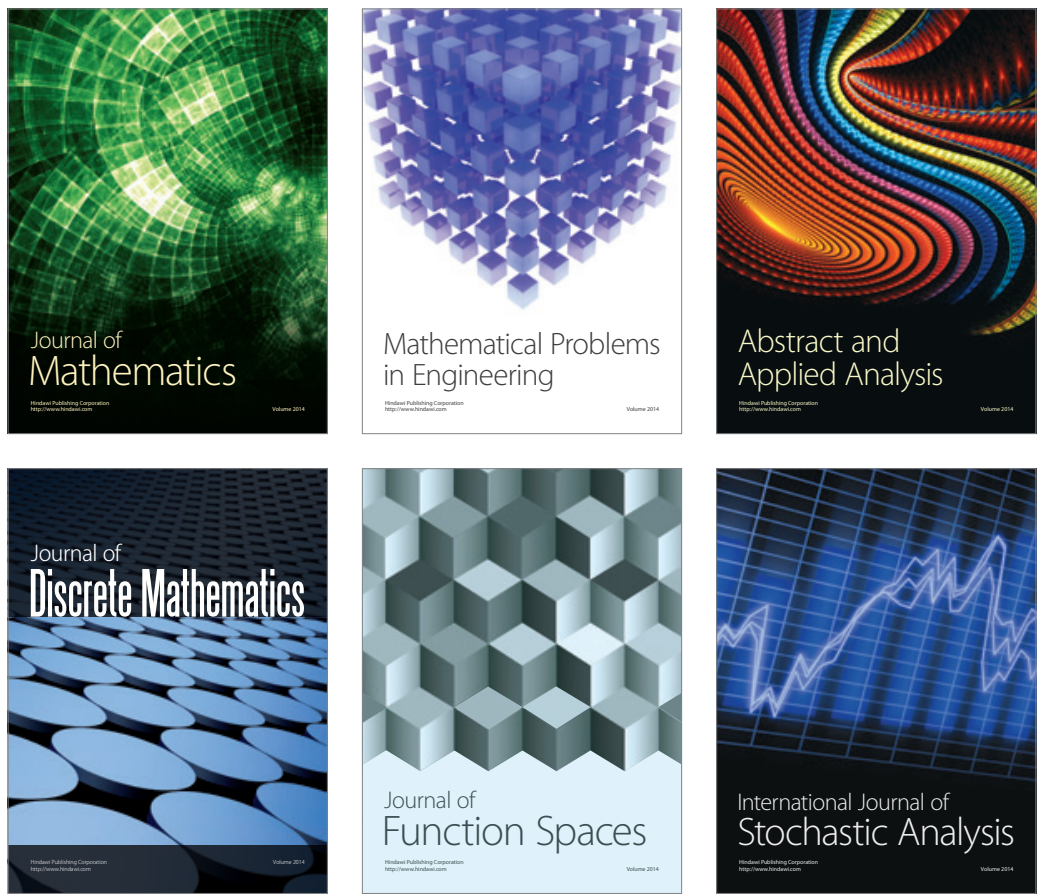

Journal of

Function Spaces

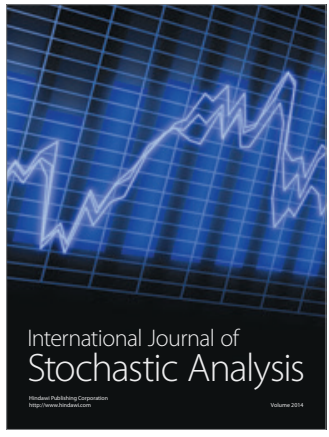

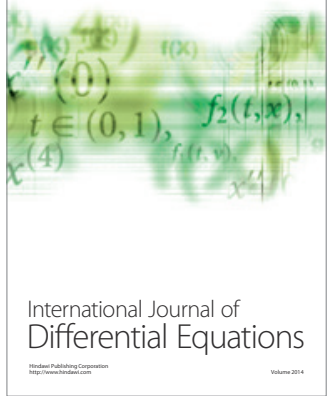
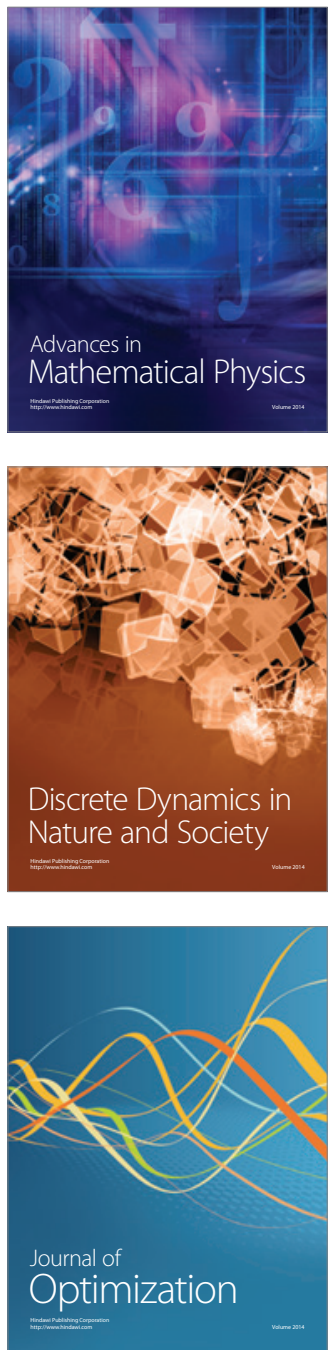